\title{
Onomástica y mentalidades en el siglo XVI
}

\author{
José ANTONIo BALlesteros Diez \\ UNED, Mérida
}

\begin{abstract}
RESUMEN
ABSTRACT

Este artículo pretende señalar que la Onomástica, entendida como un sistema simbólico, puede ser una vía de aproximación a la Historia de las Mentalidades. Se exponen unas elementales técnicas cuantitativas de análisis estadístico de series nominales correspondientes a grupos humanos determinados, y se desarrollan algunos ejemplos para mostrar cómo la Onomástica manifiesta aspectos de las mentalidades y diferencias entre las de
\end{abstract} distintos grupos sociales.

\section{LA ONOMÁSTICA COMO VIA DE APROXIMACIÓN A LAS MENTALIDADES}

Si en unos primeros momentos se acusó a la naciente Historia de las mentalidades de propiciar una nueva fragmentación en el campo de la historiografía, poco a poco, se ha ido imponiendo el hecho de que la Historia de las mentalidades, en última instancia, proporciona una nueva vía de aproximación al objetivo deseado de una historia totalizante. Y ello se comprende al ver como Peter Burke ${ }^{1}$ define la Historia de las mentalidades en relación con tres rasgos distintivos: por basarse en las actitudes colectivas más que en las individuales, y prestar tanta

\footnotetext{
1 P. BuRke: Formas de Historia cultural. Madrid, 1999, pág. 207.
} 
atención a la gente común como a los componentes de las elites; también, porque no interesan tanto las ideas conscientes, o las teorías elaboradas, como los supuestos implícitos o inconscientes; $y$, en tercer lugar, porque se dirige al conocimiento de la estructura de la creencia, además de su contenido, es decir, se interesa por cómo piensa la gente, además de conocer lo que piensa, y trata de las categorías, metáforas y símbolos en la representación que de ellos mismos transmiten, pasando de la descripción al análisis y la comprensión.

Por otra parte, también se ha superado la visión de la Historia de las mentalidades como un repertorio de hitos anclados en el tiempo, como algo ahistórico, pues, como dice Carlos Martínez Shaw ${ }^{2}$, la Historia de las mentalidades, basada en la constatación de todas las actividades humanas, analiza los gestos y las actitudes ante experiencias universales, que lejos de constituir hechos inalterables e intemporales por estar fijados desde siempre en la naturaleza humana, son realmente productos históricos porque también se modifican al mismo tiempo que los sistemas de organización social; siendo, por tanto, el conocimiento aportado por la Historia de las mentalidades fundamental para conseguir acercarnos a la historia total.

El estudio de la Onomástica ha superado su inicial vinculación con la Etimología y actualmente, al entenderse que es fundamentalmente un sistema simbólico, ha pasado a formar parte de la Historia de las mentalidades, en razón, sobre todo, del impulso de la Antropología que al reconocer el concepto de cultura como un sistema de significados, actitudes y valores compartidos, y las formas simbólicas en que se expresan o en que están insertas, ha propiciado que el estudio de los sistemas de nominación de los pueblos constituyan una valiosa fuente para aproximarnos al conocimiento de sus mentalidades y patrones de conducta, que, en este caso de la Onomástica, como ciencia dedicada a los nombres propios de personas, lugares o razas, aunque al estar preferentemente centrada en el estudio de los primeros ha dado lugar a que algunos, como Leite de Vaconcelos, la llamen Antroponímia, mientras que otros utilizan la denominación de Homonímia; aquí, en este estudio, no vamos a entrar en discutir cual es la denominación más acertada y precisa desde un punto de vista rigurosamente científico, y utilizaremos el término Onomástica por ser el más conocido, entendiéndolo como un sistema simbólico, es decir, como un producto cultural, y consecuentemente relacionado de modo directo con la Historia de las mentalidades.

La Onomástica, además del estudio etimológico de los nombres, ahora relacionado con la influencias y los préstamos culturales entre distintos pueblos, etnias y culturas a lo largo del tiempo y del espacio, nos indica también la pervivencia de tradiciones, y todo ello le proporciona una apariencia de intemporalidad, pero el histoiiador, al detectar la aparición de modas a favor de un nombre u otro, los cambios de paradigmas en las sociedades, la variación de la influencia de la religión

2 C. Martinez Shaw: "La Historia de las mentalidades", en VV.AA. (B. Casado, coordinador): Tendencias Historiográficas actuales. UNED. Madrid, 2001, pág. 305. 
por virtud de sus propios cambios, como se puede ver en la onomástica femenina del siglo XVI cuando, por causa de la Contrarreforma, aumentó considerablemente el número de niñas que fueron bautizadas con el nombre de María, a la vez que se introducían nombres inexistentes en la Edad media, como señala Américo Castro ${ }^{3}$ con los de Ascensión, Asunción, Concepción, Dolores, Angustias, Soledad, Visitación, Candelaria, Presentación, Socorro, la dispersión de distintos nombres según los movimientos migratorios, y otros muchos agentes que actúan sobre la mentalidad de las gentes, factores todos ellos que, al estudiarlos, nos demuestran cómo la asignación de un nombre a una persona no es consecuencia de un procedimiento aleatorio, $y$, por ello, el estudiar la Onomástica puede ser una vía cierta de aproximación a la Historia de las mentalidades.

La Onomástica posiblemente sea el primer sistema de símbolos utilizado en la Humanidad, pues el nombre, junto con los apellidos mucho tiempo después, constituye una forma de individualizar a una persona; porque cada nombre encierra un significado y constituye un elemento identificador, como sabemos desde los tiempos de los griegos cuando algunos filósofos sostenían que es en los nombres y no en las cosas donde radica la autenticidad, pues mediante el nombre se le da el ser a las cosas.

Una cuestión previa, muy importante, para el que estudie la onomástica de un grupo humano determinado como via de conocimiento de su mentalidad, es que ha de tener en cuenta que estos nombres sobre los que trabaja no han sido elegidos por sus propios portadores, sino, generalmente, por sus padres, abuelos o padrinos, es decir, estos nombres tenemos que reconocerlos como la expresión de una mentalidad generacionalmente anterior, que como valor medio aproximado podemos anticipar en 30 años con relación a las que estudiamos. Podriamos decir que estamos queriendo hacer hablar a quienes no se expresan.

Por otra parte, la decisión de los padres sobre el nombre que asignarán ${ }^{4}$ a sus hijos no es aleatoria, como hemos dicho, pues siempre habrá algún razonamiento que justifique esa decisión, y al estudiar la intencionalidad con que se asignan los nombres a las personas, lo debemos hacer interpretando tal hecho como un hábito, en el sentido en que lo entiende el sociólogo Pierre Bourdieu, según lo explica Bur$\mathrm{ke}^{5}$, cuando aquel se refiere a la propensión de los miembros de un grupo social a seleccionar respuestas dentro de un repertorio particular en relación a una situación concreta, pues a diferencia del concepto de regla normativa, el hábito tiene la ventaja de permitir reconocer en el que lo utiliza la disposición de un ámbito de libertad individual. Asi, mientras los apelidos muestran la pertenencia a una determinada familia, siendo muchos de ellos derivados del nombre del antepasado que encabezó

${ }^{3}$ A. CAStro: España en su historia. Barcelona, 1996, pág. 631.

${ }^{4}$ Empleo el verbo asignar, pues estimo es el que corresporide con más rigor al hecho da dar nombre a una persona, frente al uso frecuente de poner, o imponer, pues aquel alude directamente al signo, al símbolo que es en definitiva cada nombre.

${ }^{5}$ P. BuRkE: Formas de hacer Historia. Madrid, 1994, págs. 34 y 35. 
el linaje, como vemos en Rodríguez de Rodrigo, González de Gonzalo, Diaz de Diego, Sánchez de Sancho, y en tantos otros, la asignación del nombre es libre, y la adopción por lo padres del nominativo con que van a llamar a cada uno de sus hijos obedece a planteamientos más complejos, como el de mantener el suyo propio, o el de los abuelos; es decir, a esta pauta podemos considerarla como tradicional o de pervivencia de ramas dentro de cada familia; otra pauta estaba radicada en las devociones religiosas, como las debidas al santo patrón, o patrona, de la localidad, Olalla en el caso de Mérida, o Pilar en Zaragoza, que será un nombre permanente entre las mujeres a lo largo de los siglos, lo que igualmente ocurre con Rafael entre los varones de Córdoba, y así otros muchos ejemplos son posibles; también dentro de la motivación religiosa estaba la influencia que ejercían las congregaciones del clero regular desde sus conventos en las distintas localidades, y muestra de ello es que en Mérida, donde había dos conventos franciscanos, abundaba el nombre "Francisco"; la pervivencia del culto y rememoración de los mártires, presente en la Iglesia desde sus primeros tiempos, la testimonian nombres como Esteban, u Olalla; también estaban aquellos que al ser bautizados recibían como nombre el del santo del día, al que consideraban como protector del bautizado, costumbre que en Extremadura está poco extendida, al contrario de lo que se conoce sucedía en Castilla la Vieja y León, porque en Extremadura el procedimiento de asignación de un nominativo obedecía a una cierta causalidad, mientras que en esas otras regiones, para muchas personas fue la consecuencia de un procedimiento aleatorio, desde un punto de vista estadístico, pero, por el contrario, desde un punto de vista religioso, respondía a un sentido providencialista de la vida y se estimaba que ese santo del día era el protector designado por la providencia divina para los que nacieran en el día de su festividad; también, dentro de este ámbito de influencia de lo religioso, se producía la asignación del nombre tomando el del santo patrono del oficio del progenitor. La influencia religiosa, tan determinante en este siglo XVI que estudiamos, incidirá en el bautismo con la adopción del nombre de un santo al que se tiene una persona devoción, y que, por encima de esta, respondía además al deseo de ver realizadas en una persona ciertas representaciones ideales de una determinada forma de vida que muestra también sus propias variaciones, y así, en España, se reflejará en la onomástica la fuerte corriente contrarreformista, o del catolicismo barroco, lo que se empezará a manifestar a partir del Concilio de Trento, es decir, en las décadas finales de la centuria, momentos en que también se introducen nuevas fiestas en la calendario católico, como la de la Virgen del Rosario, en 1571, por la victoria cristiana en la batalla de Lepanto.

Otra fuente de nombres es la que producía la influencia política, es decir, cuando los vasallos adoptaban para sus hijos el nombre de su señor jurisdiccional, en tierras de carácter señorial; lo que también se había producido en los bautismos de niños moriscos a los que se asignaba el nombre del propietario para el que trabajaban sus padres; también es posible que se produjera una influencia en todo el reino con los nombres de los miembros de la familia real, aunque con una incidencia mínima, como vemos con la los nombres de Felipe o Carlos, los primeros 
monarcas de la dinastía de los Austrias en España; y, por supuesto, también habría modas en el uso de nombres que se extenderían más o menos lentamente por todo el reino sin que hubiese una explicación inmediata al hecho de adoptar esos nuevos nombres. En este aspecto de las modas, ya en el año 1535 se quejaba Juan de Valdés de que la lengua castellana había tomado muchos vocablos de la arábiga, a pesar de que "para muchas cosas de las que nombramos con vocablos arábigos tenemos vocablos latinos, pero el uso nos ha hecho tener por mejores los arábigos que los latinos" ${ }^{6}$, sin que hubiera una causa que justificara esta predilección por lo que el humanista castellano consideraba ajeno frente a lo propio.

Había nombres que eran utilizados casi exclusivamente por algunas minoría o grupos determinados, como sucedía en Mérida durante la segunda mitad del siglo XVI con el nombre de Bernabé, muy utilizado, pero casi únicamente por los moriscos. Sin embargo, los conversos generalmente utilizaban un nombre, e incluso apellidos, distintos de los de sus antecesores para evitar la relación con la anterior religión de la familia.

Las migraciones interiores también llevaban consigo la transferencia de nombres entre localidades o territorios diferentes, y así tenemos, por ejemplo, en Vicente y Antonio unos nombres de clara procedencia portuguesa.

Por todo ello, la asignación del nombre como identificativo de una persona, no es una mera cuestión lingüística, o la expresión de un sistema simbólico, sino un hecho social bastante complejo que, en gran medida, respondia a una mentalidad, por lo que los nombres han estado siempre en evolución, como ya en esa centuria señalaba Juan de Valdés; de ahí que estudiar la onomástica de una población sea una manera de acercarse al conocimiento de algunos elementos característicos de su mentalidad colectiva y a su evolución en el tiempo y en el espacio, manifestada por los cambios de uso de unos nombres frente a otros, con lo cual tropezamos con el gran problema que Roger Chartier ${ }^{7}$ ve en el estudio de la Historia de las mentalidades, que es el de conocer y comprender las razones y las formas de paso de un sistema a otro, cuestión ciertamente compleja, pues como punto de partida en el problema tenemos el propio concepto de mentalidad, como resultado de la interacción de sentimientos, actitudes y símbolos, y también de cómo se expresan y manifiestan en opiniones o expresiones verbales, lo que puede resultar lleno de ambigüedad. Por ello, para no presentar la comprensión de las mentalidades como un ideal inalcanzable, es prudente y realista ir avanzando con pasos cortos y en ámbitos muy determinados, como es el estudio de la onomástica de unos grupos humanos a lo largo del tiempo, tratando de aproximarnos a las causas que han inducido cambios o diferencias en unos respecto a otros, buscando conocer por qué emergen nuevos nombres y también por que otros desaparecen, $y$ este estudio ha de iniciarse mediante un análisis cuantitativo del conjunto de nombres, siguiendo unas técnicas bastante sencillas que seguidamente exponemos.

\footnotetext{
6 J. de Valdés: Diálogo de la lengua. Madrid, 2003, pág. 134.

7 P. BURKE: Formas de historia..., pág. 218.
} 


\section{ELEMENTOS DE ANÁLISIS CUANTITATIVO}

En primer lugar, una vez elegido el grupo a analizar, relacionaremos todos los nombres y determinaremos la frecuencia de cada uno de ellos, que expresaremos porcentualmente, pudiéndose hacer esta determinación de frecuencias relativas sobre todo el conjunto, es decir, incluyendo mujeres y hombres, o sobre cada uno de los subconjuntos que determina el sexo de las personas cuyos nombres estudiamos, siendo esta la forma más habitual de verse en los estudios de Onomástica, aunque, en mi opinión, se deben hacer los dos. También es conveniente determinar porcentualmente el número de nombres del conjunto que sólo son utilizados por una persona, así como también los que han sido asignados a varias. Como ejemplo de este primer paso veremos un conjunto de bautizados durante la primera mitad del siglo $\mathrm{xv}^{8}$, que corresponden a una parroquia de Mérida:

Nombres de bautizados (Primera mitad siglo Xvi)

\begin{tabular}{lcc|lcc}
\hline \multicolumn{1}{c}{$\begin{array}{c}\text { Nombres } \\
\text { masculinos }\end{array}$} & Frecuencia & $\begin{array}{c}\text { Frecuencia } \\
\text { relativa }\end{array}$ & $\begin{array}{c}\text { Nombres } \\
\text { femeninos }\end{array}$ & Frecuencia & $\begin{array}{c}\text { Frecuencia } \\
\text { relativa }\end{array}$ \\
\hline Francisco & 83 & $18,9 \%$ & María & 130 & $28,9 \%$ \\
Juan & 76 & $17,4 \%$ & Isabel & 57 & $12,7 \%$ \\
Alonso & 51 & $11,6 \%$ & Catalina & 46 & $10,2 \%$ \\
Pedro & 31 & $7,1 \%$ & Juana & 32 & $7,1 \%$ \\
Hernando & 25 & $5,7 \%$ & Ana & 26 & $5,8 \%$ \\
Diego & 23 & $5,3 \%$ & Leonor & 26 & $5,8 \%$ \\
Cristóbal & 22 & $5,0 \%$ & Francisca & 26 & $5,8 \%$ \\
Rodrigo & 17 & $3,9 \%$ & Beatriz & 20 & $4,4 \%$ \\
Gonzalo & 13 & $3,0 \%$ & Inés & 20 & $4,4 \%$ \\
Bartolomé & 10 & $2,3 \%$ & Elvira & 17 & $3,8 \%$ \\
García & 9 & $2,1 \%$ & Olalla & 9 & $2,0 \%$ \\
Álvaro & 7 & $1,6 \%$ & Teresa & 8 & $1,8 \%$ \\
Miguel & 6 & $1,4 \%$ & Lucia & 4 & $0,9 \%$ \\
Luis & 5 & $1,1 \%$ & Costanza & 4 & $0,9 \%$ \\
Andrés & 5 & $1,1 \%$ & Mencia & 4 & $0,9 \%$ \\
Gómez & 5 & $1,1 \%$ & Florencia & 3 & $0,7 \%$ \\
Martín & 5 & $1,1 \%$ & Marina & 3 & $0,7 \%$ \\
Lope & 4 & $0,9 \%$ & Aldonza & 2 & $0,4 \%$ \\
Esteban & 3 & $0,7 \%$ & Martina & 2 & $0,4 \%$ \\
Blas & 3 & $0,7 \%$ & Casta & 2 & $0,4 \%$ \\
Mateo & 5 & $0,7 \%$ & Estebania & 1 & $0,2 \%$ \\
\hline Lón & 5 & & & &
\end{tabular}

8 J. A. Ballesteros Diez: «Bautismos e historia social en Mérida a principios del siglo XVI». Revista de Estudios Extremeños, Tomo LVII, Núm. II (Badajoz, 2001), págs. 647-660. Los datos de este estudio demográfico, realizado por el método de la agrupación por familias, se tomaron de los registros contenidos en el Libro I de Bautismos de la parroquia de Santa María, de Mérida, y comprende los años de 1526 a 1539 . 
Nombres de bautizados (Primera mitad siglo xvI)

(continuación)

\begin{tabular}{|c|c|c|c|c|c|}
\hline $\begin{array}{l}\text { Nombres } \\
\text { masculinos }\end{array}$ & Frecuencia & $\begin{array}{c}\text { Frecuencia } \\
\text { relativa }\end{array}$ & $\begin{array}{l}\text { Nombres } \\
\text { femeninos }\end{array}$ & Frecuencia & $\begin{array}{l}\text { Frecuencia } \\
\text { relativa }\end{array}$ \\
\hline Gabriel & 3 & $0,7 \%$ & Narbona & 1 & $0,2 \%$ \\
\hline Manuel & 2 & $0,5 \%$ & Clara & 1 & $0,2 \%$ \\
\hline Lorenzo & 2 & $0,5 \%$ & Sabina & 1 & $0,2 \%$ \\
\hline Tomé & 2 & $0,5 \%$ & Elena & 1 & $0,2 \%$ \\
\hline Melchor & 2 & $0,5 \%$ & Mayor & 1 & $0,2 \%$ \\
\hline Jerónimo & 2 & $0,5 \%$ & Blanca & 1 & $0,2 \%$ \\
\hline Gaspar & 2 & $0,5 \%$ & Luisa & 1 & $0,2 \%$ \\
\hline Gutierre & 2 & $0,5 \%$ & Andrea & 1 & $0,2 \%$ \\
\hline Pascual & 1 & $0,2 \%$ & & & \\
\hline Antonio & 1 & $0,2 \%$ & & & \\
\hline Ambrosio & 1 & $0,2 \%$ & & & \\
\hline Vasco & 1 & $0,2 \%$ & & & \\
\hline Fabián & 1 & $0,2 \%$ & & & \\
\hline Domingo & 1 & $0,2 \%$ & & & \\
\hline Felipe & 1 & $0,2 \%$ & & & \\
\hline Toribio & 1 & $0,2 \%$ & & & \\
\hline Matías & 1 & $0,2 \%$ & & & \\
\hline Tello & 1 & $0,2 \%$ & & & \\
\hline Antón & 1 & $0,2 \%$ & & & \\
\hline Juan de Ortega & 1 & $0,2 \%$ & & & \\
\hline Serván & 1 & $0,2 \%$ & & & \\
\hline Asensio & 1 & $0,2 \%$ & & & \\
\hline Pablo & 1 & $0,2 \%$ & & & \\
\hline Baltasar & 1 & $0,2 \%$ & & & \\
\hline \multicolumn{3}{|c|}{438} & & 450 & \\
\hline
\end{tabular}

En la tabla hemos ordenado los nombres en orden decreciente de frecuencias, y de ello evidentemente se obtienen ya algunas conclusiones, pero antes de seguir en la interpretación de estos datos, debemos continuar el análisis cuantitativo determinado otros parámetros que nos ayudarán en la comprensión de estos conjuntos de nombres, y para ello seguimos la metodología explicada por Antonio Carretero ${ }^{9}$. En primer lugar tenemos el «Índice de Dispersión», (ID), que nos relaciona el número de nombres distintos asignados a las personas del grupo y el numero de éstas que los componen:

Índice de Dispersión: $\quad I D=\Sigma$ nombres $/ \Sigma$ personas

9 A. Carretero Melo: “Onomástica y Demografia. Apuntes metodológicos». Nouvelle revue d,Onomastique, N. ${ }^{\circ}$ 39-40 (Paris, 2002), págs. 221-237. 
Para dos conjunto con igual número de personas, el ID mayor nos indica que ese conjunto de nombres es numericamente mayor que el del grupo que tiene ID menor, y representa el número de nombres disponibles por persona.

Otro índices el denominado «Promedio Isonímico», (PI), que es precisamente el inverso del anterior

\section{Promedio Isonímico: $\quad \mathrm{PI}=\Sigma$ personas $/ \Sigma$ nombres}

que representa el número medio de personas que corresponden a cada nombre.

Otro índice es el que sive para medir la relación entre el número total de nombres y el número de nombres que están asignados a una sóla persona, es el llamado «Índice de Nominación Única», (INU), el cual se calcula dividiendo el número de nombres de única nominación por el total de nombres.

Índice Nominación Única: $\quad I N U=\Sigma$ nombres nominación única $/ \Sigma$ nombres

Como estos índices están muy influenciados por el tamaño de la muestra, para corregir esa influencia y poder hacer comparaciones más válidas entre grupos desiguales, se utiliza el denominado "Índice de Isonimia" (II), que se calcula tomado la cifra de nombres usados por más de una persona, elevado al cuadrado, y dividiendo por el cuadrado del número total de nombres. Este índice sive para determinar la probabilidad de que una persona del grupo, elegida al azar, tenga asignado el mismo nombre que otra.

Índice Isonimia: $\quad I I=\Sigma$ (nombres nominación múltiple $)^{2} / \Sigma$ (nombres) $^{2}$

Como práctica aplicaremos estos parámetros a los dos conjuntos de nombres que hemos visto anteriormente, y que mostramos en la tabla siguiente:

Indices estadísticos

\begin{tabular}{lcc}
\hline \multicolumn{1}{c}{ Concepto } & Niñas & Niños \\
\hline - Número de nombres & 29 & 46 \\
- Número de nombres usados por una sóla persona & $9(31,03 \%)$ & $16(34,78 \%)$ \\
- Número de nombres de nominación múltiple & $20(68,97 \%)$ & $30(65,22 \%)$ \\
- Número de personas & 450 & 438 \\
- ID (Índice de dispersión) & 0,064 & 0,105 \\
- PI (Promedio isonímico) & 15,5 & 9,5 \\
- INU (Índice de nominación única) & 0,31 & 0,35 \\
- II (Índice de isonimia) & 0,475 & 0,425 \\
\hline
\end{tabular}

Si ahora tomamos el conjunto global de personas, sin dividirlos por sexos, obtenemos una nueva tabla de frecuencias, que para no alargar el texto exponemos simplemente en la representación gráfica de los 20 nombres con mayor frecuencia de asignación: 


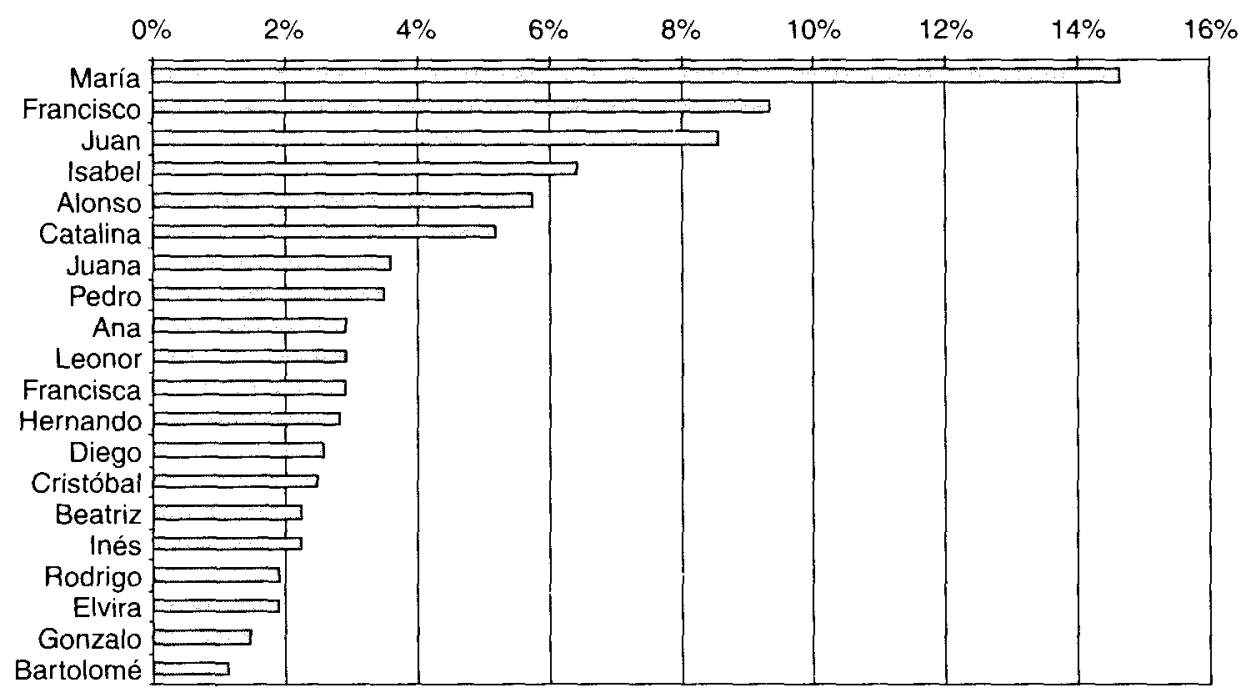

Frecuencia relativa de los nombres más usados.

Como vemos, hay un nombre femenino que destaca en su frecuencia de asignación notoriamente sobre todos los demás, tanto femeninos como masculinos, aparte de la interpretación simbólica que se pueda hacer, aquí sólo vamos a señalar que esta frecuencia tan alta supone una reducción del número de los otros nombres asignados a las mujeres, cuyo ID es 0,064 frente al 0,105 del de los hombres. Los valores estadísticos de esta tabla conjunta son los siguientes:

\begin{tabular}{lc}
\hline \multicolumn{1}{c}{ Concepto } & Valores \\
\hline - Número de nombres & 75 \\
- Número de nombres usados por una sóla persona & $25(33,33 \%)$ \\
- Número de nombres de nominación múltiple & $50(66,67 \%)$ \\
- Número de personas & 888 \\
- ID (Índice de dispersión) & 0,084 \\
- PI (Promedio isonímico) & 11,84 \\
- INU (Índice de nominación única) & 0,333 \\
- II (Índice de isonímia) & 0,444 \\
\hline
\end{tabular}

\section{LA ONOMÁSTICA DURANTE EL SIGLO XVI}

Para tener una referencia de partida, vamos a tomar los resultados de un estudio sobre registros nominales de la segunda mitad del siglo $\mathrm{XV}$, realizado por $\mathrm{M}^{\mathrm{a}}{ }^{\mathrm{a}}$ del Carmen Aguilera sobre documentos del Archivo Provincial Histórico de Córdoba $^{10}$, en el cual vemos que el nombre femenino más asignado es Maria $(14,3 \%)$,

$10 \mathrm{M} \cdot{ }^{\text {a }} \mathrm{C}$. Aguilera Castro: "Los nombres de los cordobeses en la segunda mitad del siglo XV". ACtas del VI Curso de cultura medieval, sobre la vida cotidiana en la España Medieval. Aguilar de Campoo. Palencia, 1994. 
seguido de Catalina (13,4\%), Isabel $(10,8 \%)$ y Leonor (10,1\%); a continuación aparecen Marina, Juana, Beatriz, Inés, Teresa, Elvira, Costanza, Lucía, Ana, Antonia, Mencia, Mayor, Urraca, Luisa, Cristina, Bartolomeba, Aldonza, Sancha, Bernardina, Victoria, Florinda, Elena, Águeda, Clarencia, Angelina y Francisca; en total, estos treinta nombres los llevaban el $86,1 \%$ de las mujeres cordobesas. En cuanto a los varones, Juan era el nombre más usado $(23,2 \%)$, seguido de Pedro $(13,8 \%)$, Alfonso $(12,4 \%)$, Antonio $(8,3 \%)$, Fernando $(6 \%)$, Diego $(5,7 \%)$ y después seguían Gonzalo, Martin, Bartolomé, Rodrigo, Luis, Francisco, Miguel, Andrés y Lope; estos quince nombres los tenian asignado el $91,3 \%$ de los hombres.

A la vista de esta referencia, si recordamos los nombres de los Reyes Católicos y los de sus hijos: Isabel, Juan, Juana, María y Catalina, comprobamos que están entre los más utilizados, lo que nos indica, en este caso, que a pesar de ser estos Reyes las figuras estelares de su tiempo, no era la sociedad la que recogía sus nombres, sino ellos los que asumían la misma mentalidad que caracterizaba a la sociedad castellana en la prelación de unos nombres respecto de otros. Es decir, no parece que hubiera influencia política en la nominación de los castellanos.

Por razón de la disponibilidad de documentos, las bases de datos utilizadas para este estudio corresponden a la población de Extremadura, y como tratamos de ver la relación entre onomástica y mentalidad a lo largo del tiempo, veremos separadamente los datos correspondientes a las dos mitades de la centuria y para la primera parte disponemos de los registros bautismales de la parroquia de Santa María de Mérida para los años comprendidos entre 1526 y 1539 y además la relación de los integrantes de la expedición de Hernando de Soto a la Florida, en el año $1538^{11}$, la cual nos va a permitir conocer las diferencias entre los nombres de los extremeños y los de otros castellanos miembros de la hueste, formando todos un conjunto bastante homogéneo en cuanto a las edades y condiciones sociales.

Arrancamos conociendo los nombres asignados a las niñas al ser bautizadas en Mérida, y la relación, en orden decreciente, estaba encabezada por María $(38,9 \%)$, como vemos un porcentaje muy superior al de Córdoba, que, en principio podemos interpretar como una muestra de distinta mentalidad regional, por efecto del paso del tiempo, o por la conjunción de ambas causas. Seguidamente aparecen los nombres de Isabel (12,7\%), Catalina (10,2\%), Juana $(7,1 \%)$, Ana, Leonor y Francisca (el $5,8 \%$ cada una), y a continuación los de Beatriz, Inés, Elvira, Olalla, Teresa, Lucía, Costanza, Mencía, Florencia, Marina, Aldonza, Martina, Casta, Estebanía, Narbona, Clara, Sabina, Elena, Mayor, Blanca, Luisa y Andrea, en total 29 nombres que se aplicaron sobre el $100 \%$ de las niñas bautizadas. Si comparamos este conjunto de nombres emeritenses con los de los cordobeses del siglo XV, vemos que en ambos conjuntos es María el nombres más veces asignado, y ocupa pues el primer lugar en la ordenación de mayor a menor frecuencia de los nombres; pero observamos que en Mérida, ya en siglo xvI, la frecuencia del nombre

11 R. Sánchez Rubio: “Extremeños con Hernando de Soto en la expedición a la Florida". Actas del Congreso de Hernando de Soto y su tiempo. Badajoz, 1993, págs. 17-51. 
María es el doble de la que había en Córdoba, lo cual nos indica un avance considerable de la devoción mariana en la religiosidad popular; los dos nombres siguientes también coinciden en ambas relaciones, aunque invertido el orden; estos tres nombres ocupan en Mérida la mitad de la relación de niñas bautizadas.

La relación de los nombres asignados a los varones en los bautismos de Mérida nos da un total de 45 nombres, y en primer lugar aparece el de Francisco, que fue asignado al $18,9 \%$ de los bautizados, seguido por Juan $(17,4 \%)$, Alonso $(11,6 \%)$, Pedro $(7,1 \%)$, Hernando $(5,7 \%)$ y Diego $(5,3 \%)$; comparando con la relación de nombres cordobeses vemos una notable diferencia, pues, recordemos, éstos los encabezaban Juan $(23,2 \%)$, Pedro $(13,8 \%)$, Alfonso (12,4\%), Antonio $(8,3 \%)$, Fernando $(6 \%)$ y Diego $(5,7 \%)$, que ocupaban los seis primeros lugares. Las diferencias son notables, pues Francisco en Córdoba era un nombre minoritario, mientras en Mérida era el más usado; con Juan, Pedro, Alonso, Hernando y Diego hay bastante coincidencia, aunque los porcentajes de nominación sean dispares, y, además, en la relación cordobesa aparece extendido Antonio mientras que ese nombre en Mérida solamente era usado por una persona.

Considerando el factor de reproducción de la identidad de los padres sobre los hijos, tenemos que en Mérida, en esta primera mitad del siglo xVI, el $9,1 \%$ de los niños repiten el nombre de su padre, mientras el $5,9 \%$ de las niñas lo hacen con el de su madre; de los niños, el 0,3\% llevan el nombre de su madre, y de las niñas el $0,1 \%$ son bautizadas con el nombre de su padre; por lo tanto, para todo el conjunto de bautizados, el 9,2 repiten el nombre de su padre y el $6,2 \%$ el de su madre; es decir, el deseo de reproducir la identidad paterna es un $50 \%$ superior al de la materna.

La otra relación de nombres es la de los expedicionarios a la Florida, que seguidamente detallamos, distinguimos entre extremeños, no extremeños y los bautizados en Mérida, con cuyos datos formamos la tabla siguiente:

Nombres de varones en la primera mitad del siglo XVI

\begin{tabular}{|c|c|c|c|c|c|c|}
\hline \multirow[b]{2}{*}{$\begin{array}{l}N{ }^{\circ} \text { de } \\
\text { orden }\end{array}$} & \multicolumn{2}{|c|}{ Extrremeños } & \multicolumn{2}{|c|}{ No extremeños } & \multicolumn{2}{|c|}{ Emeritenses } \\
\hline & Nombre & $\begin{array}{c}\text { Frecuencia } \\
(\%)\end{array}$ & Nombre & $\begin{array}{c}\text { Frecuencia } \\
(\%)\end{array}$ & Nombre & $\begin{array}{c}\text { Frecuencia } \\
(\%)\end{array}$ \\
\hline 10 & Juan & 20,46 & Juan & 22,78 & Francisco & 18,9 \\
\hline $2 .^{\circ}$ & Pedro & 11,82 & Francisco & 11,39 & Juan & 17,4 \\
\hline 3. & Francisco & 10,09 & Pedro & 11,39 & Alonso & 11,6 \\
\hline $4 .^{\circ}$ & Alonso & 8,93 & Diego & 7,28 & Pedro & 7,1 \\
\hline $5 .^{\circ}$ & Diego & 6,05 & Alonso & 6,96 & Hernando & 5,7 \\
\hline $6 .^{\circ}$ & Hernando & 4,32 & Bartolomé & 4,11 & Diego & 5,3 \\
\hline $7 .^{\circ}$ & Gonzalo & 4,03 & Aritonio & 2,85 & Cristóbal & 5,0 \\
\hline $8 .^{\circ}$ & Andrés & 3,46 & Cristóbal & 2,85 & Rodrigo & 3,9 \\
\hline $9 .^{\circ}$ & Álvaro & 3,46 & Luis & 2,22 & Gonzalo & 3,0 \\
\hline $10^{\circ}$ & Bartolomié & 2,88 & Andrés & 1,90 & Bartolomé & 2,3 \\
\hline
\end{tabular}


Nombres de varones en la primera mitad del siglo XVI (continuación)

\begin{tabular}{|c|c|c|c|c|c|c|}
\hline \multirow[b]{2}{*}{$\begin{array}{l}N .^{\circ} \text { de } \\
\text { orden }\end{array}$} & \multicolumn{2}{|c|}{ Extrremeños } & \multicolumn{2}{|c|}{ No extremeños } & \multicolumn{2}{|c|}{ Emeritenses } \\
\hline & Nombre & $\begin{array}{c}\text { Frecuencia } \\
(\%)\end{array}$ & Nombre & $\begin{array}{l}\text { Frecuencia } \\
\text { (\%) }\end{array}$ & Nombre & $\begin{array}{c}\text { Frecuencia } \\
\text { (\%) }\end{array}$ \\
\hline $11 .^{\circ}$ & Rodrigo & 2,02 & Martín & 1,90 & García & 2,1 \\
\hline $12 .^{\circ}$ & García & 1,44 & Miguel & 1,27 & Álvaro & 1,6 \\
\hline $13^{\circ}$ & Cristóbal & 1,15 & Fernán & 1,27 & Miguel & 1,4 \\
\hline $14 .^{\circ}$ & Luis & 1,15 & Hernando & 1,27 & Luis & 1,1 \\
\hline $15 .^{\circ}$ & Gómez & 1,15 & Agustín & 0,95 & Andrés & 1,1 \\
\hline $16 .^{\circ}$ & Manuel & 1,15 & Bernaldo & 0,95 & Gómez & 1,1 \\
\hline $17 .^{\circ}$ & Bernaldo & 0,86 & Gaspar & 0,95 & Martín & 1,1 \\
\hline $18^{\circ}$ & Lope & 0,86 & Melchor & 0,95 & Lope & 0,9 \\
\hline $19^{\circ}$ & Esteban & 0,86 & Gonzalo & 0,95 & Esteban & 0,7 \\
\hline $20 .^{\circ}$ & Simón & 0,86 & Toribio & 0,95 & Blas & 0,7 \\
\hline $21^{\circ}$ & Fabián & 0,86 & García & 0,95 & Mateo & 0,7 \\
\hline $22 .^{\circ}$ & Benito & 0,86 & Lucas & 0,95 & Gabriel & 0,5 \\
\hline $23 .^{\circ}$ & Vasco & 0,86 & Rodrigo & 0,95 & Manuel & 0,5 \\
\hline $24 .^{\circ}$ & Antonio & 0,58 & Bautista & 0,63 & Lorenzo & 0,5 \\
\hline $25 .^{\circ}$ & Martín & 0,58 & Gómez & 0,63 & Tomé & 0,5 \\
\hline $26 .^{\circ}$ & Fernán & 0,58 & Gutierre & 0,63 & Melchor & 0,5 \\
\hline $27 .^{\circ}$ & Domingo & 0,58 & Domingo & 0,63 & Jerónimo & 0,5 \\
\hline $28 .^{\circ}$ & Baltasar & 0,58 & Jerónimo & 0,63 & Gaspar & 0,5 \\
\hline $29 .^{\circ}$ & Sebastián & 0,58 & Baltasar & 0,63 & Gutierre & 0,5 \\
\hline $30 .^{\circ}$ & Lorenzo & 0,58 & Arias & 0,63 & Pascual & 0,2 \\
\hline $31 .{ }^{\circ}$ & Carlos & 0,58 & Gregorio & 0,63 & Antonio & 0,2 \\
\hline $32 .^{\circ}$ & Pablo & 0,58 & Sebastián & 0,63 & Ambrosio & 0,2 \\
\hline $33 .^{\circ}$ & Alejo & 0,58 & Lázaro & 0,63 & Vasco & 0,2 \\
\hline $34 .^{\circ}$ & Miguel & 0,29 & Bonifacio & 0,32 & Fabián & 0,2 \\
\hline $35 .^{\circ}$ & Gaspar & 0,29 & Salvador & 0,32 & Domingo & 0,2 \\
\hline $36 .^{\circ}$ & Lucas & 0,29 & Mateo & 0,32 & Felipe & 0,2 \\
\hline $37 .^{\circ}$ & Jerónimo & 0,29 & Lope & 0,32 & Matías & 0,2 \\
\hline $38^{\circ}$ & Arias & 0,29 & Lorenzo & 0,32 & Tello & 0,2 \\
\hline $39 .^{\circ}$ & Bernabé & 0,29 & Manuel & 0,32 & Antón & 0,2 \\
\hline $400^{\circ}$ & Antón & 0,29 & Arrio & 0,32 & Juan de Ortega & 0,2 \\
\hline $41^{\circ}$ & Pascual & 0,29 & Santiago & 0,32 & Serván & 0,2 \\
\hline $42 .^{\circ}$ & Mateo & 0,29 & Ruy & 0,32 & Asensio & 0,2 \\
\hline $43 .^{\circ}$ & Jorge & 0,29 & Aparicio & 0,32 & Pablo & 0,2 \\
\hline $44 .^{\circ}$ & Marcos & 0,29 & Tristán & 0,32 & Baltasar & 0,2 \\
\hline $45 .^{\circ}$ & Mendo & 0,29 & Julián & 0,32 & & \\
\hline $46 .^{\circ}$ & Salvador & 0,29 & Bernabé & 0,32 & & \\
\hline $47 .^{\circ}$ & Atanasio & 0,29 & Esteban & 0,32 & & \\
\hline $48 .^{\circ}$ & Nuño & 0,29 & Vicente & 0,32 & & \\
\hline $49 .^{\circ}$ & Blas & 0,29 & Giraldo & 0,32 & & \\
\hline $50 .^{\circ}$ & & & Simón & 0,32 & & \\
\hline $51 .^{\circ}$ & & & Cósimo & 0,32 & & \\
\hline
\end{tabular}


Los parámetros estadísticos para los nombres de la expedición a La Florida, los detallamos seguidamente haciendo separación entre extremeños y castellanos no extremeños:

\begin{tabular}{lcc}
\hline \multicolumn{1}{c}{ indices } & Extremeños & $\begin{array}{c}\text { Castellanos } \\
\text { no extremeños }\end{array}$ \\
\hline $\mathrm{N} .{ }^{\circ}$ nombres diferentes & 51 & 49 \\
$\mathrm{~N} .{ }^{\circ}$ de personas & 316 & 347 \\
$\mathrm{~N} .{ }^{\circ}$ de nombres usados sólo por una persona & $18(35,29 \%)$ & $16(32,65 \%)$ \\
$\mathrm{N} .^{\circ}$ de nombres usados por varias personas & $33(64,71 \%)$ & $33(67,35 \%)$ \\
Dispersión & 16,14 & 14,12 \\
$\mathrm{PI}$ & 6,2 & 7,08 \\
INU & 35,29 & 32,65 \\
II & 0,419 & 0,454 \\
\hline
\end{tabular}

Hemos marcado en negrita los nombres que aparecen repetidos en las tres relaciones, y aunque haya una variable diferencia de extensión del uso de cada uno de ellos, representan evidentemente una coincidencia general; es en los nombres que sólo aparecen en una o en dos listas, donde vamos a fijarnos para aproximarnos a esa diferencia de mentalidad que pueden representar; el más llamativo es Álvaro, que ocupa el puesto $9 .^{\circ}$ entre los extremeños y el $12 .^{\circ}$ entre los emeritenses, sin que aparezca entre los otros castellanos, como tampoco lo hacia entre los de Córdoba, lo que los asemeja a los castellanos no extremeños de la relación de la Florida, entre los cuales, por cierto, había una mayoría de andaluces. Álvaro, como se sabe, es un nombre de origen germánico introducido en Hispania por los visigodos, igual que Elvira entre los femeninos, y su presencia en Extremadura puede estar relacionada con el proceso de repoblación siguiente a la Reconquista, que en el solar extremeño fue ocupado en algunas áreas por los monarcas leoneses mientras en otras lo hacian los castellanos, y una característica de aquellos era que ejercian la justicia mediante la aplicación estricta de normas o códigos escritos, mientras que los castellanos seguían normas consuetudinarias en cada uno de los lugares; la diferencia es evidentemente muy grande eritre una y otra práctica judicial, y revela en los leoneses la fidelidad a la tradición visigótica, mientras en Castilla se olvidan de todo ese cuerpo normativo y lo dejan al buen sentido de los jueces de cada lugar. Es decir, entre los del Reino de León hay una fuerte permanencia de la mentalidad visigótica, que, al menos en estos aspectos, está ausente entre los castellanos; quizá también radique en esta mentalidad el hecho de que la Orden de Santiago llame a sus territorios en la actual Extremadura como «Provincia de León", mientras que los asentados en la Mancha, Murcia o Andalucía constituyan la "Provincia de Castilla"; otro testimonio de esta pervivencia en Extremadura de la tradición gótica la tenemos en el uso del nombre "Alonso, que había sido introducido en España por los godos y significa primero o principal, derivado de la letra griega alfa" (Covarrubias), y que vemos en la onomástica extremeña con mayor presencia que en la no extremeña. Para no extender en ex- 
ceso el comentario de similitudes y diferencias entre estas listas, y por ser poco significativos cuantitativamente los nombres que no coinciden en una o dos de ellas, dejamos en este punto el comentario de la tabla, no sin antes llamar la atención de cómo algunos nombres aparecen en dos formas distintas, Pedro y Simón; Diego y Santiago, pues no hay que olvidar que Diego se formó por derivación de las formas primeras: Sant Yago, Yago, Diago, Diego (Covarrubias). Y como ausencia llamativa para nuestra onomástica actual está el nombre de José.

Para la segunda mitad de la centuria contamos con más fuentes y, algunas, numéricamente mayores. En primer lugar, las de Mérida ${ }^{12}$, donde los bautismos de la Parroquia de Santa María cubren ya la totalidad de este período, y también contamos con los de la otra parroquia emeritense en aquel siglo, la de Santa Olalla, cuyos registros se conservan desde 1580. Con respecto a estas nominaciones, tomaremos en primer lugar las de los bautismos de la parroquia de Santa María para comparar, y enlazar, con los datos que hemos visto de la primera mitad del siglo, puesto que este nuevo conjunto de nominaciones corresponde en continuidad a la misma población, por lo que las diferencias que observemos serán de bastante interés. Las relaciones de nombres, con sus frecuencias relativas, las exponemos en la tabla siguiente:

Nombres de bautizados en Sta. M. ${ }^{a}$ (Mérida) entre 1550 y 1600

\begin{tabular}{|c|c|c|c|c|}
\hline \multirow[b]{2}{*}{$N^{\circ}$ de orden } & \multicolumn{2}{|c|}{ Niños } & \multicolumn{2}{|c|}{ Niñas } \\
\hline & Nombres & Frecuencia (\%) & Nombres & Frecuencia (\%) \\
\hline 1. & Juan & 25,7 & María & 41,0 \\
\hline $2 .^{\circ}$ & Francisco & 13,4 & Isabel & 10,9 \\
\hline 3.0 & Alonso & 8,8 & Catalina & 10,1 \\
\hline $4 .^{\circ}$ & Diego & 7,6 & Ana & 6,4 \\
\hline $5 .^{\circ}$ & Pedro & 7,3 & Juana & 5,6 \\
\hline $6 .^{\circ}$ & Hernando & 4,3 & Leonor & 3,6 \\
\hline $7 .^{\circ}$ & Cristóbal & 3,2 & Beatriz & 3,6 \\
\hline $8^{\circ}$ & Andrés & 2,4 & Francisca & 3,2 \\
\hline $9 .^{\circ}$ & Antonio & 2,2 & Inés & 3,0 \\
\hline $10{ }^{\circ}$ & Sebastián & 1,9 & Olalla & 2,6 \\
\hline $11^{\circ}$ & Miguel & 1,7 & Elvira & 2,4 \\
\hline $12 .^{\circ}$ & Rodrigo & 1,6 & Mencia & 1,7 \\
\hline $13^{\circ}$ & Luis & 1,5 & Teresa & 1,0 \\
\hline $14 .^{\circ}$ & Bartolomé & 1,5 & Marina & 0,7 \\
\hline $15^{\circ}$ & Gonzalo & 1,3 & Mayor & 0,4 \\
\hline $16^{\circ}$ & Comingo & 1,1 & Antonia & 0,4 \\
\hline $17 .^{\circ}$ & Gaspar & 1,0 & Costanza & 0,4 \\
\hline $18 .^{\circ}$ & Lorenzo & 1,0 & Jerónima & 0,3 \\
\hline $19 .^{\circ}$ & Martín & 0,9 & Lucía & 0,3 \\
\hline $200^{\circ}$ & Serván & 0,9 & Madalena & 0,2 \\
\hline $21 . \circ$ & García & 0,8 & Guiomar & 0,2 \\
\hline $22 .^{\circ}$ & Álvaro & 0,7 & Luisa & 0,2 \\
\hline $23^{\circ}$ & Marcos & 0,7 & Estebanía & 0,1 \\
\hline
\end{tabular}

12 J. A. Ballesteros Diez: “Bautismos, confirmaciones y matrimonios en la historia social de Mérida en la segunda mitad del siglo XV|'. Revista de Estudios Extremeños, Tomo LVIII, Núm. III (Badajoz, 2002), págs. 941-990. 
Nombres de bautizados en Sta. M. ${ }^{a}$ (Mérida) entre 1550 y 1600 (continuación)

\begin{tabular}{|c|c|c|c|c|}
\hline \multirow[b]{2}{*}{ N. de orden } & \multicolumn{2}{|c|}{ Niños } & \multicolumn{2}{|c|}{ Niñas } \\
\hline & Nombres & Frecuencia (\%) & Nombres & Frecuencia (\%) \\
\hline $24 .^{\circ}$ & Mateo & 0,6 & Aldonza & 0,1 \\
\hline $25 .^{\circ}$ & Lope & 0,5 & Brígida & 0,1 \\
\hline $26 .^{\circ}$ & Joseph & 0,4 & Florencia & 0,1 \\
\hline $27 .^{\circ}$ & Tomé & 0,4 & Melchora & 0,1 \\
\hline $28 .^{\circ}$ & Melchor & 0,4 & Mariana & 0,1 \\
\hline $29 .^{\circ}$ & Blas & 0,4 & Dominga & 0,1 \\
\hline $30 .^{\circ}$ & Antón & 0,3 & Elena & 0,1 \\
\hline $31 .{ }^{\circ}$ & Gabriel & 0,3 & Marta & 0,1 \\
\hline $32 .^{\circ}$ & Fabián & 0,3 & Bernardina & 0,1 \\
\hline $33 .^{\circ}$ & Bernabé & 0,3 & Marcela & \\
\hline $34 .^{\circ}$ & Manuel & 0,3 & Victoria & \\
\hline $35 .^{\circ}$ & Benito & 0,3 & Gracia & \\
\hline $36 .^{\circ}$ & Vasco & 0,3 & Brazayda & \\
\hline $37 .^{\circ}$ & Bernardo & 0,3 & Bárbara & \\
\hline $38 .^{\circ}$ & Gómez & 0,3 & Violante & \\
\hline $39 .^{\circ}$ & Agustín & 0,2 & Ida & \\
\hline $400^{\circ}$ & Lucas & 0,2 & Sara & \\
\hline $41 .^{\circ}$ & Lázaro & 0,2 & Felipa & \\
\hline $42 .^{\circ}$ & Baltasar & 0,2 & Germana & \\
\hline $43 .^{\circ}$ & Simón & 0,2 & Juliana & \\
\hline $44 .^{\circ}$ & Esteban & 0,2 & Clara & \\
\hline $45^{\circ}$ & Felipe & 0,1 & Martina & \\
\hline $46 .^{\circ}$ & Juan Francisco & 0,1 & María de la Cruz & \\
\hline $47 .^{\circ}$ & Ambrosio & 0,1 & Blanca & \\
\hline $48 .^{\circ}$ & Toribio & 0,1 & Servana & \\
\hline $499^{\circ}$ & Jerónimo & 0,1 & Jacobina & \\
\hline $50 .^{\circ}$ & Pascual & 0,1 & Lorena & \\
\hline $51 .^{\circ}$ & Juan Antonio & 0,1 & M. ${ }^{a}$ Encarnación & \\
\hline $52 .^{\circ}$ & Tomás & 0,1 & Ángela & \\
\hline $53 .^{\circ}$ & Juan Luis & & Quiteria & \\
\hline $54 .^{\circ}$ & Isidoro & & Cristina & \\
\hline $55 .^{\circ}$ & Pablo & & & \\
\hline $56 .^{\circ}$ & Jorge & & & \\
\hline $57 .^{\circ}$ & Miguel Jerónimo & & & \\
\hline $58 .^{\circ}$ & Nicolás & & & \\
\hline $59 .^{\circ}$ & Gregorio & & . & \\
\hline $60 .^{\circ}$ & Tadeo & & & \\
\hline $61 .^{\circ}$ & Zebrián & & & \\
\hline $62 .^{\circ}$ & Aparicic & & & \\
\hline $63 .^{\circ}$ & Roque & & & \\
\hline $64 .^{\circ}$ & Ginés & & & \\
\hline $65 .^{\circ}$ & Francisco Jacinto & & & \\
\hline $66 .^{\circ}$ & Dionisio & & & \\
\hline $67 .^{\circ}$ & Juan Baltasar & & & \\
\hline $68 .^{\circ}$ & Jacinto & & & \\
\hline $69 .^{\circ}$ & Alfonso & & & \\
\hline $700^{\circ}$ & Julián & & & \\
\hline $71 .^{\circ}$ & Matías & & & \\
\hline $72 .^{\circ}$ & Salvador & & & \\
\hline
\end{tabular}


Desde un punto de vista cuantitativo, lo primero que apreciamos es el aumento considerable del número de nombres utilizados, que detallamos en el cuadro siguiente con la comparación de los datos de igual población en la primera mitad de la centuria:

Bautismos en Sta. Maria (Mérida) durante el siglo XVI

\begin{tabular}{l|cc|cc}
\hline \multirow{2}{*}{\multicolumn{1}{c|}{ Índices }} & \multicolumn{2}{|c|}{ Primera mitad del siglo } & \multicolumn{2}{c}{ Segunda mitad del siglo } \\
\cline { 2 - 5 } & Niñas & Niños & Niñas & Niños \\
\hline N. ${ }^{\circ}$ nombres & 29 & 45 & 54 & 72 \\
Nombres usados por más de una persona & $20(69 \%)$ & $29(64 \%)$ & $32(59,3 \%)$ & $52(72 \%)$ \\
Nombres usados sólo por una persona & $9(31 \%)$ & $16(36 \%)$ & $22(40,7 \%)$ & $20(28 \%)$ \\
Dispersión & 0,064 & 0,105 & 0,024 & 0,031 \\
PI & 15,5 & 9,5 & 40,89 & 32,35 \\
INU & 0,31 & 0,35 & 0,400 & 0,278 \\
II & 0,475 & 0,425 & 0,360 & 0,562 \\
\hline
\end{tabular}

Si hacemos un paralelismo con el parámetro demográfico de la Razón de masculinidad, estableceremos la relación entre el número de nombres masculinos y el de los femeninos, que para la primera mitad del siglo será 1,55 y para la segunda 1,31 , es decir, aunque han aumentado considerablemente los nombres, este incremento ha sido más importante para el grupo femenino que para el masculino, aunque este siga siendo mayor que el otro. En cuando al uso de los nombres por una o por varias personas, vemos cómo entre las niñas se acentúa la tendencia a la individualización, pues los nombres unipersonales pasan en su asignación del $31 \%$ al $40 \%$, mientras que entre los varones se produce el fenómenos contrario, pues del $36 \%$ de nombres unipersonales de la primera parte se pasa al $28 \%$ en la segunda, lo que se puede interpretar como una disminución de la individualización entre los varones.

Si comparamos la ordenación de los nombres de los niños de la primera mitad del siglo con la de la segunda vemos cómo se están produciendo unas alteraciones considerables, el primer lugar que ocupaba Francisco ahora corresponde a Juan, que to hace con mas de la cuarta parte del total de los nominados, descendiendo notablemente Francisco, como también lo hacen Rodrigo, Gonzalo, Bartolomé..., y, recíprocamente, vemos la aparición entre los primeros nombres de Andrés, Antonio, Sebastián... De Álvaro se observa una considerable disminución, plies pasa del $1,6 \%$ al $0,7 \%$, síntoma quizá de la castellanización de la sociedad emeritense; y Bernabé, que no estaba presente en la primera mitad de la centuria ahora representa el $0,3 \%$, que aunque es una participación muy pequeña es, sin embargo, muy significativa, porque todos los que llevaban ese nombre eran moriscos, y esta etnia tuvo en Mérida una implantación considerable al recibirse en la 
ciudad a muchos de los moriscos expulsados de las tierras granadinas tras la guerra de las Alpujarras.

Observando las relaciones nominales de las niñas, resalta que María es el nombre más usado, no sólo entre éstas sino también considerando el conjunto de niños, ningún nombre se aproximaba al de María en la asignación a los bautizados, además el crecimiento es ciertamente altísimo, pues pasa del 28,9 al $41 \%$, lo que manifiesta el resultado del adoctrinamiento contrarreformista que se producía en España; los nombres que siguen son los mismos de la primera mitad, con ligeros cambios en el ordenamiento; el nombre de Olalla, la mártir patrona de la ciudad, aumenta su implantación del $2 \%$ al 2,6\%, lo que manifiesta el aumento de la devoción popular hacia una de las primeras santas del cristianismo hispano.

Como ya hicimos con los datos de la primera mitad del siglo para la población de esta misma parroquia, agruparemos los registros de niñas y niños y mostraremos en un gráfico la variación que han tenido las frecuencias relativas que vimos en la primera mitad respecto de las de la segunda:

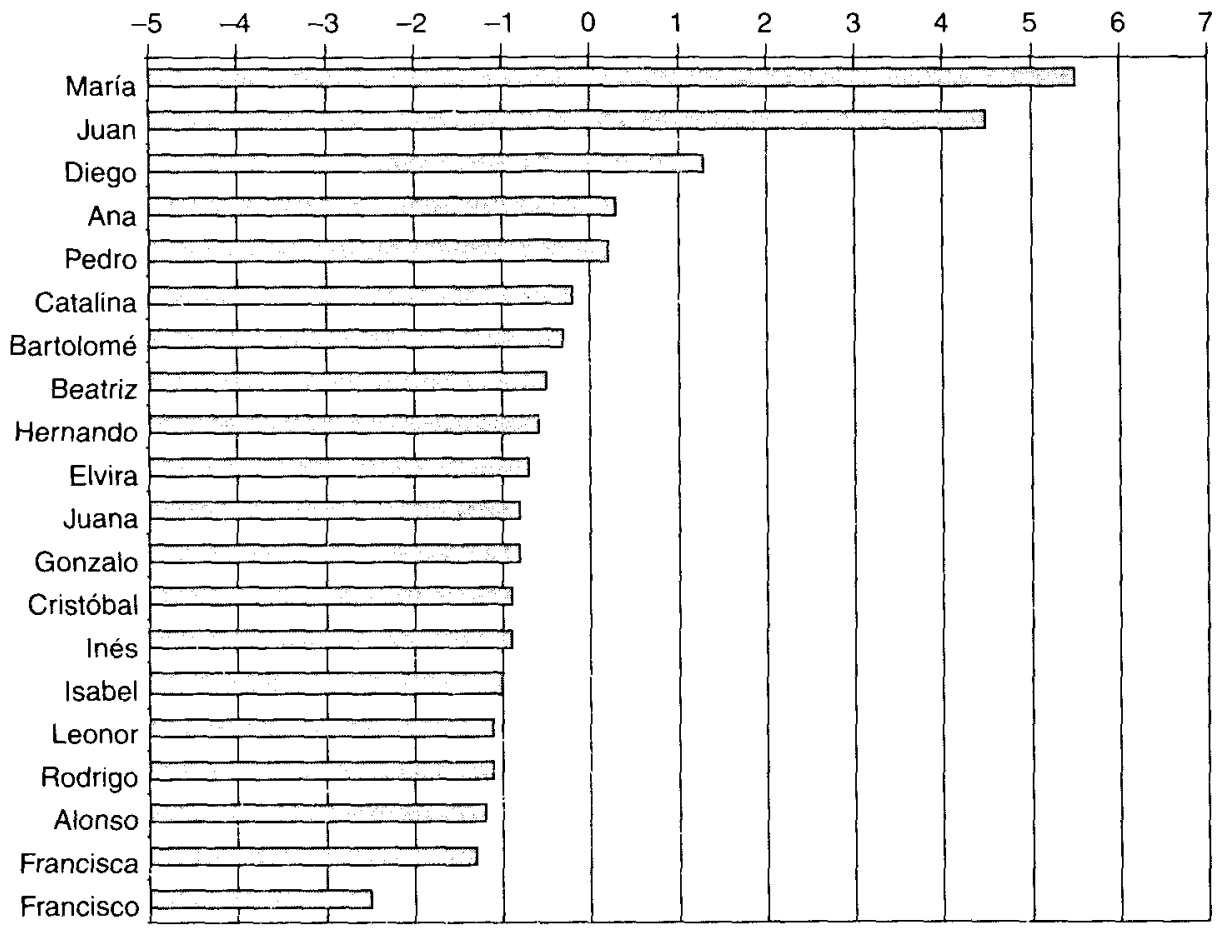

Variación $1 .^{a}$ mitad-2. mitad.

Como vemos, los nombres Maria, Juan, Diego, Ana y Pedro, aumentan su implantación, mientras que los demás disminuyen. 
Para terminar las nominaciones bautismales emeritenses, consideramos las de la parroquia de Santa Olalla, que comprenden las dos últimas décadas de la centuria y que mostramos en la siguiente relación:

Nombres de bautizados en Sta. Olalla (Mérida) entre 1580 y 1600

\begin{tabular}{|c|c|c|c|c|}
\hline \multirow[b]{2}{*}{$N .^{\circ}$ de orden } & \multicolumn{2}{|c|}{ Niños } & \multicolumn{2}{|c|}{ Niñas } \\
\hline & Nombres & Frecuencia (\%) & Nombres & Frecuencia (\%) \\
\hline $1 .{ }^{\circ}$ & Juan & 29,3 & María & 42,5 \\
\hline $2 .^{\circ}$ & Francisco & 12,3 & Isabel & 11,7 \\
\hline $3 .^{\circ}$ & Alonso & 9,0 & Ana & 9,0 \\
\hline $4 .^{\circ}$ & Pedro & 8,5 & Catalina & 8,5 \\
\hline $5 .^{\circ}$ & Diego & 7,2 & Juana & 6,0 \\
\hline $6 .^{\circ}$ & Bartolomé & 3,0 & Olalla & 4,5 \\
\hline $7 .^{\circ}$ & Miguel & 2,9 & Leonor & 2,5 \\
\hline $8 .^{\circ}$ & Fernando & 2,8 & Beatriz & 2,5 \\
\hline $9 .^{\circ}$ & Antonio & 2,8 & Inés & 2,2 \\
\hline $10^{\circ}$ & Cristóbal & 2,4 & Francisca & 2,1 \\
\hline $11^{\circ}$ & Andrés & 1,9 & Elvira & 1,9 \\
\hline $12 .^{\circ}$ & Sebastián & 1,9 & Marina & 0,8 \\
\hline $13^{\circ}$ & Domingo & 1,3 & Lucía & 0,7 \\
\hline $14 .^{\circ}$ & Martín & 1,1 & Mencía & 0,6 \\
\hline $15^{\circ}$ & Lorenzo & 1,0 & Estebanía & 0,4 \\
\hline $16^{\circ}$ & Gaspar & 1,0 & Antonia & 0,4 \\
\hline $17 .^{\circ}$ & Tomé-Tomás & 1,0 & Madalena & 0,4 \\
\hline $18 .^{\circ}$ & García & 0,9 & Jerónima & 0,4 \\
\hline $19 .^{\circ}$ & Rodrigo & 0,9 & Luisa & 0,3 \\
\hline $20 .^{\circ}$ & Luis & 0,8 & Jacinta & 0,2 \\
\hline $21 .^{\circ}$ & Gonzalo & 0,7 & Guiomar & 0,2 \\
\hline $22 .^{\circ}$ & Baltasar & 0,5 & Angela & 0,2 \\
\hline $23 .^{\circ}$ & Manuel & 0,5 & Florencia & 0,2 \\
\hline $24 .^{\circ}$ & Fabián & 0,5 & Teresa & 0,2 \\
\hline $25 .^{\circ}$ & Blas & 0,5 & Rufina & 0,1 \\
\hline $26 .^{\circ}$ & Bernabé & 0,4 & Bárbara & 0,1 \\
\hline $27 .^{\circ}$ & José & 0,4 & Mayor & 0,1 \\
\hline $28 .^{\circ}$ & Mateo & 0,3 & Cecilia & 0,1 \\
\hline $29 .^{\circ}$ & Marcos & 0,3 & Águeda & 0,1 \\
\hline 30. & Lázaro & 0,3 & Andrea & 0,1 \\
\hline $31 .^{\circ}$ & Serván & 0,3 & Agustina & 0,1 \\
\hline $32 .^{\circ}$ & Felipe & 0,3 & Bernardina & 0,1 \\
\hline $33^{\circ}$ & Simón & 0,2 & Clara & 0,1 \\
\hline $34 .^{\circ}$ & Gabriel & 0,2 & Costanza & 0,1 \\
\hline $35^{\circ}$ & Álvaro & 0,2 & Elena & 0,1 \\
\hline $36 .^{\circ}$ & Benito & 0,2 & Gracia & 0,1 \\
\hline $37 .^{\circ}$ & Bernardo & 0,2 & Juliana & 0,1 \\
\hline $38^{\circ}$ & Pascual & 0,2 & Lorenza & 0,1 \\
\hline $39 .{ }^{\circ}$ & Esteban & 0,2 & María Antonia & 0,1 \\
\hline
\end{tabular}


Nombres de bautizados en Sta. Olalla (Mérida) entre 1580 y 1600 (continuación)

\begin{tabular}{|c|c|c|c|c|}
\hline \multirow[b]{2}{*}{ N. ${ }^{\circ}$ de orden } & \multicolumn{2}{|c|}{ Niños } & \multicolumn{2}{|c|}{ Niñas } \\
\hline & Nombres & Frecuencia (\%) & Nombres & Frecuencia (\%) \\
\hline $40{ }^{\circ}$ & Toribio & 0,2 & María Francisca & 0,1 \\
\hline $41^{\circ}$ & Jerónimo & 0,2 & Petronila & 0,1 \\
\hline $42 .^{\circ}$ & Salvador & 0,2 & Sebastiana & 0,1 \\
\hline $43 .^{\circ}$ & Gregorio & 0,1 & Violante & 0,1 \\
\hline $44 .^{\circ}$ & Agustín & 0,1 & & \\
\hline $45 .^{\circ}$ & Antón & 0,1 & & \\
\hline $46 .^{\circ}$ & Jacinto & 0,1 & & \\
\hline $47 .^{\circ}$ & Lope & 0,1 & & \\
\hline $48 .^{\circ}$ & Lucas & 0,1 & & \\
\hline $49 .^{\circ}$ & Matías & 0,1 & & \\
\hline $50 .^{\circ}$ & Nicolás & 0,1 & & \\
\hline $51 .^{\circ}$ & Santiago & 0,1 & & \\
\hline
\end{tabular}

El análisis cuantitativo lo resumimos en el siguiente cuadro:

Bautizados en Sta. Olalla (Mérida) entre 1580 y 1600

\begin{tabular}{lcc}
\hline \multicolumn{1}{c}{ Índices } & Niñas & Niños \\
\hline N. ${ }^{\circ}$ nombres & 43 & 51 \\
N. de personas & 1.217 & 1.239 \\
Nombres usados por más de una persoria & $24(55,8 \%)$ & $42(82,4 \%)$ \\
Nombres usados sólo por una persona & $19(44,2 \%)$ & $9(17,6 \%)$ \\
Dispersión & 0,036 & 0,041 \\
PI & 47,65 & 24,29 \\
INU & 0,442 & 0,176 \\
II & 0,323 & 0,678 \\
\hline
\end{tabular}

El que estos datos correspondan a las dos últimas décadas del siglo nos puede proporcionar información acerca de las distintas tendencias que hemos atisbado en las relaciones anteriores comprensivas de toda la segunda mitad, y entre ellas venos cómo la razón entre el número de nombres masculinos y el de femeninos es de 1,18, lo que manifiesta el acercamiento entre ambas relaciones, también se confirma la mayor propensión a la individualidad entre las niñas que entre los niños, siendo aquí todavia más acusado este hecho que en Santa Maria, pues el número de nombres unipersonales alcanza en las niñas al $44,2 \%$, y en los niños desciende al $17,6 \%$. 
Al comparar las relaciones nominales vemos que, en la de los niños, los cinco primeros son coincidentes, salvo un cambio de orden, y que los porcentajes muestran una gran correlación. En Santa Olalla vemos una mayor presencia del nombre Bernabé, por razón de que esa parroquia se concentró una mayor parte de la población morisca inmigrada, y que aquí alcanza al 0,40, frente al $0,3 \%$ de Santa María; también hay aquí una menor presencia de Álvaro, el 0,2 frente al $0,7 \%$. José, un nombre emergente, aparece igualado en las dos parroquias, con el $0,4 \%$. Con respecto a los nombres de las niñas, María sigue siendo el primero, también el más utilizado entre todos los bautizados de ambos sexos, y en Santa Olalla su participación es del 42,5\%; el nombre de Olalla, en esta parroquia que lleva su nombre, es el $6 .^{\circ}$ y lo llevan el $4,5 \%$ de las niñas.

Si consideramos la totalidad de las nominaciones efectuadas en Mérida durante la segunda mitad del siglo, y en relación a la continuidad de los nombres de los progenitores en sus hijos, tenemos que el $13,9 \%$ de los niños llevan el nombre de su padre, y el $0,7 \%$ el de su madre, mientras que el $10,3 \%$ de las niñas repite el nombre de su madre y el $0,6 \%$ el de su padre; por lo que, en conjunto, el nombre del padre es aplicado sobre el $14,5 \%$ y el de la madre sobre el $11 \%$. Es evidente la disminución de estos porcentajes respectos a los que determinamos para la primera mitad del siglo, y este mismo hecho confirma la tendencia a la mayor individualización que hemos detectado; lo que, en alguna medida, representa la asimilación por la mentalidad popular de la revalorización de la persona que había promovido el humanismo renacentista.

Antonio Carretero ${ }^{13}$, en un estudio sobre los nombres de pila de los varones bautizados en La Fuente del Maestre, entre los años 1554 y 1719, al analizar la influencia familiar en la nominación, ha determinado por décadas la coincidencia del nombre del padre con el del hijo, encontrando que el valor más bajo se produjo entre los años $1550-1560$, con el $23 \%$, y el más alto entre $1610-1620$ con el $32,4 \%$; como vemos porcentajes muy superiores a los de Mérida y con una tendencia opuesta, pues en esta localidad tiende al crecimiento mientras en Mérida lo hace hacia la disminución. También distingue el comportamiento de los vecinos oriundos de la villa con respecto a los llegados forasteros, y nos dice que, entre los primeros, la influencia paterna es débil sobre los dos primeros hijos, mientras que entre los forasteros es mayor, y que el tercer hijo era el preferido para recibir el nombre del progenitor.

El haber realizado un estudio demográfico de Mérida por el procedimiento de la agrupación por familias, nos permite aplicar a este trabajo algunas de las observaciones allí obtenidas para poder diferenciar la onomástica utilizada por la población cristiana (vieja) frente a la de los moriscos (cristianos nuevos), aunque sólo daremos el detalle de los diez primeros lugares:

13 A. Carretero Melo: “Antroponimia en la Edad Moderna. El nombre de pila del varón». Nouvelle revue d'Onomastique (Socreté francaise d'Onomastique). N. ${ }^{\circ} 37-38$ (Paris, 2001), págs. 43-56. 
Comparación cristianos viejos frente a moriscos en Mérida

\begin{tabular}{|c|c|c|c|c|c|c|c|c|}
\hline \multirow{3}{*}{ N. de orden } & \multicolumn{4}{|c|}{ Niñas } & \multicolumn{4}{|c|}{ Niños } \\
\hline & \multicolumn{2}{|c|}{ Cristianos viejos } & \multicolumn{2}{|c|}{ Moriscos } & \multicolumn{2}{|c|}{ Cristianos viejos } & \multicolumn{2}{|c|}{ Moriscos } \\
\hline & Nombre & $(\%)$ & Nombre & $(\%)$ & Nombre & $(\%)$ & Nombre & $(\%)$ \\
\hline $1 .^{\circ}$ & Maria & 40,5 & María & 45,1 & Juan & 26,3 & Juan & 20,3 \\
\hline $2 .^{\circ}$ & Catalina & 11,0 & Isabel & 26,3 & Francisco & 14,5 & Alonso & 13,5 \\
\hline $3 .^{\circ}$ & Isabel & 9,2 & Beatriz & 6,3 & Alonso & 8,2 & Miguel & 11,8 \\
\hline $4 .^{\circ}$ & Ana & 6,9 & Inés & 3,1 & Pedro & 8,0 & Diego & 6,8 \\
\hline $5 .^{\circ}$ & Juana & 5,9 & Juana & 2,7 & Diego & 7,7 & Hernando & 6,8 \\
\hline $6 .^{\circ}$ & Leonor & 3,8 & Leonor & 2,2 & Hernando & 4,0 & Andrés & 5,1 \\
\hline $7 .^{\circ}$ & Francisca & 3,5 & Lucía & 2,2 & Cristóbal & 3,6 & Bernabé & 5,1 \\
\hline $8 .^{\circ}$ & Beatriz & 3,4 & Catalina & 1,8 & Antonio & 2,3 & Lorenzo & 4,6 \\
\hline $9 .^{\circ}$ & Inés & 2,9 & Elvira & 1,8 & Andrés & 2,1 & Luis & 4,6 \\
\hline $10^{\circ}$ & Olalla & 2,8 & Ana & 1,3 & Sebastián & 2,0 & Francisco & 3,8 \\
\hline
\end{tabular}

Durante el siglo XVI estaba muy vivo el problema de la asimilación religiosa de los moriscos por lo que, al bautizarse, solian adquirir nombres muy relacionados con la religiosidad popular y las devociones de cada lugar, por ello no es sorprendente que los nombres de María e Isabel fueran más utilizados por los moriscos que por los cristianos viejos, para parecerse lo más posible a la población dominante; posiblemente el de Maria por la corriente religiosa contrarreformista, pero en el de Isabel, además de la tradicional tendencia, es posible fuese incrementada por la memoria, ya legendaria en esos tiempos, de la reina Isabel; con lo que los moriscos conjugarian tanto influencias religiosas como políticas en su lógico afán de supervivencia dentro del reino castellano; y así también encontrarnos con el nombre de Olalla entre la población morisca femenina, que aumentó mucho en Mérida durante el último tercio del siglo. Comparando datos de bautismos de niñas hijas de cristianos viejos y los de hijas de moriscos, tenemos la siguiente tabla:

\section{El nombre Olalla entre moriscos y cristianos en Mérida}

\begin{tabular}{lcc}
\hline \multicolumn{1}{c}{ Bautismos en Santa María } & Hijas de cristianos viejos & Hijas de moriscos \\
\hline - Número de nombres empleados & 54 & 21 \\
- Número de Olallas & 109 & 5 \\
- Relación: N. ${ }^{\circ}$ Olalla $/ \mathrm{N} .{ }^{\circ}$ de nombres & $1,85 \%$ & $4,76 \%$ \\
\hline
\end{tabular}

Entre los varones también es Juan, el nombre más empleado, igual que entre los cristianos, pero el nombre Berriabé, como ya hemos dicho, no fue asignado a ningún niño hijo de cristianos viejos y, sin embargo, lo llevaban el $5,1 \%$ de los niños moriscos; también el nombre Álvaro, que entre los cristianos supone el 0,7 entre los moriscos es el 1,3\%; por el contrario, Pedro, que en los cristianos nomina al $8 \%$ de los niños, entre los moriscos solamente lo hace sobre el 1,7\%, y con Francisco estos porcentajes son, respectivamente, $14,5 \%$ y $3,8 \%$. 
Otra colectividad de la que hemos dispuesto es la de los ilegítimos bautizados en Cáceres, sobre cuya documentación trabajó Ángel Rodriguez ${ }^{14}$, recopilando las relaciones siguientes:

llegítimos en Cáceres en la segunda mitad del siglo xvI

\begin{tabular}{|c|c|c|c|c|}
\hline \multirow[b]{2}{*}{ N. ${ }^{\circ}$ de orden } & \multicolumn{2}{|c|}{ Niños } & \multicolumn{2}{|c|}{ Niñas } \\
\hline & Nombres & Frecuencia (\%) & Nombres & Frecuencia (\%) \\
\hline $1 .^{\circ}$ & Juan & 23,4 & María & 38,3 \\
\hline $2 .^{\circ}$ & Francisco & 14,6 & Isabel & 10,9 \\
\hline $3 .^{\circ}$ & Antonio & 7,6 & Ana & 8,6 \\
\hline $4 .^{\circ}$ & Diego & 6,4 & Catalina & 6,9 \\
\hline $5 .^{\circ}$ & Alonso & 4,7 & Francisca & 5,7 \\
\hline $6 .^{\circ}$ & Pedro & 4,1 & Juana & 4,6 \\
\hline $7 .^{\circ}$ & Jerónimo & 4,1 & Inés & 4,0 \\
\hline $8 .^{\circ}$ & Benito & 2,3 & Elena & 1,7 \\
\hline $9 .^{\circ}$ & Cristóbal & 2,3 & Elvira & 1,7 \\
\hline $10 .^{\circ}$ & Gabriel & 2,3 & Benita & 1,7 \\
\hline $11 .^{\circ}$ & Luis & 1,8 & Mencia & 1,7 \\
\hline $12{ }^{\circ}$ & Hernando & 1,8 & Madalena & 1,1 \\
\hline $13 .^{\circ}$ & Agustin & 1,8 & Jerónima & 1,1 \\
\hline $14 .^{\circ}$ & Pablo & 1,8 & Paula & 1,1 \\
\hline $15^{\circ}$ & Antón & 1,2 & Lucia & 1,1 \\
\hline $16 .^{\circ}$ & Mateo & 1,2 & Pascuala & 1,1 \\
\hline $17 .^{\circ}$ & Manuel & 1,2 & Teresa & 1,1 \\
\hline $18 .^{\circ}$ & Ambrosio & 1,2 & Leonor & 1,1 \\
\hline $19 .^{\circ}$ & Sebastián & 1,2 & Petrona & 0,6 \\
\hline $20 .^{\circ}$ & Domingo & 1,2 & Bárbara & 0,6 \\
\hline $21 .^{\circ}$ & Jorge & 1,2 & Victoria & 0,6 \\
\hline $22 .^{\circ}$ & Andrés & 1,2 & Casilda & 0,6 \\
\hline $23 .^{\circ}$ & Martín & 1,2 & Polonia & 0,6 \\
\hline $24 .^{\circ}$ & Bartolomé & 1,2 & Beatriz & 0,6 \\
\hline $25 .^{\circ}$ & Lucas & 1,2 & Felicitas & 0,6 \\
\hline $26 .^{\circ}$ & Joseph & 0,6 & Brigida & 0,6 \\
\hline $27 .^{\circ}$ & Lorenzo & 0,6 & Ostanza & 0,6 \\
\hline $28 .^{\circ}$ & Sancho & 0,6 & Antonia & 0,6 \\
\hline $29 .{ }^{\circ}$ & Bertol & 0,6 & Clara & 0,6 \\
\hline $30.0^{\circ}$ & Gonzalo & 0,6 & & \\
\hline $31 . \circ$ & Gregorio & 0,6 & & \\
\hline $32{ }^{\circ}$ & Damián & 0,6 & & \\
\hline $33 .^{\circ}$ & Baltasar & 0,6 & & \\
\hline $34 !^{\circ}$ & Félix & 0,6 & & \\
\hline $35 .^{\circ}$ & Álvaro & 0,6 & & \\
\hline $36 .^{\circ}$ & Macías & 0,6 & & \\
\hline $37 .{ }^{\circ}$ & Máximo & 0,6 & & \\
\hline $38 .^{\circ}$ & Felipe & 0,6 & & \\
\hline $39 .{ }^{\circ}$ & Blas & 0,6 & & \\
\hline
\end{tabular}

14 A. Rodriguez SánChez: "La natalidad ilegítima en Cáceres durante el siglo XVI". Revista de Estudios Extremeños. Tomo XXXV, Núm. I (Badajoz, 1979), págs. 125-164. Aunque el trabajo comprende todo el siglo, sin embargo, la mayoría de los registros corresponden a su segunda mitad, y son estos datos los que hemos seleccionado para el presente estudio. 
El resumen cuantitativo es el siguiente:

Bautismos en Cáceres de ilegítimos

\begin{tabular}{|c|c|c|}
\hline Indices & Niñas & Niños \\
\hline N. nombres & 29 & 39 \\
\hline N. ${ }^{\circ}$ de personas & 175 & 171 \\
\hline Nombres usados por más de una persona & $18(62 \%)$ & $25(64 \%)$ \\
\hline Nombres usados sólo por una persona & $11(38 \%)$ & $14(36 \%)$ \\
\hline Dispersión & 15,67 & 22,81 \\
\hline $\mathrm{PI}$ & 6,03 & 4,38 \\
\hline INU & 0,379 & 0,36 \\
\hline II & 0,385 & 0,41 \\
\hline
\end{tabular}

Como vemos, aquí se muestra una tendencia semejante en cuanto a la individualización de la persona por el uso de nombre tanto en los niños como entre las niñas. Tampoco aparece el nombre Bernabé entre los varones, y es que solamente se reseñan como pertenecientes a la etnia morisca a dos madres, a cuyos tres hijos bautizaron respectivamente con los nombres de Francisca, Diego y Juana.

Otra base de datos disponible es la de los extremeños que emigraron a Indias, cuyos viajes fueron controlados individualmente por la Casa de Contratación ${ }^{15}$, y su relación nominal es la siguiente:

Extremeños pasajeros a Indias en el siglo XVI

\begin{tabular}{c|lr|lc}
\hline \multirow{2}{*}{$N$} & \multicolumn{2}{|c|}{ Varones } & \multicolumn{2}{c}{ Mujeres } \\
\cline { 2 - 5 }.$^{\circ}$ de orden & \multicolumn{2}{|c|}{ Nombres } & Frecuencia (\%) & \multicolumn{2}{c}{ Nombres } & Frecuencia (\%) \\
\hline $1 .^{\circ}$ & Juan & 17,0 & María & 21,1 \\
$2 .^{\circ}$ & Francisco & 12,0 & Catalina & 11,0 \\
$3 .^{\circ}$ & Alonso & 11,7 & Isabel & 10,6 \\
$4 .^{\circ}$ & Pedro & 9,3 & Inés & 8,6 \\
$5 .^{\circ}$ & Diego & 7,6 & Juana & 7,4 \\
$6 .^{\circ}$ & Hernando & 5,9 & Beatriz & 6,7 \\
$7 .^{\circ}$ & Gonzalo & 4,4 & Elvira & 6,7 \\
$8 .^{\circ}$ & Rodrigo & 2,3 & Ana & 5,9 \\
$9 .^{\circ}$ & Martín & 2,2 & Leonor & 3,9 \\
$10^{\circ}$ & Bartolomé & 1,8 & Teresa & 3,1 \\
$11 .^{\circ}$ & Antón & 1,8 & Francisca & 2,7 \\
$12 .^{\circ}$ & Cristóbal & 1,7 & Mencía & 2,0 \\
$13 .^{\circ}$ & García & 1,7 & Elena & 1,2 \\
\hline
\end{tabular}

15 Relación tomada de Internet (www:nobiliaria.com/apellidosconq/), donde se relacionan, por apellidos, los pasajeros extremeños a las Indias durante el siglo xvl, aunque solamente relaciona los apellidos cuya inicial está comprendida entre A y J. 
Extremeños pasajeros a Indias en el siglo xVI (continuación)

\begin{tabular}{|c|c|c|c|c|}
\hline \multirow[b]{2}{*}{ N. ${ }^{\circ}$ de orden } & \multicolumn{2}{|c|}{ Varones } & \multicolumn{2}{|c|}{ Mujeres } \\
\hline & Nombres & Frecuencia (\%) & Nombres & Frecuencia (\%) \\
\hline $14^{\circ}$ & Andrés & 1,3 & Ostanza & 1,2 \\
\hline $15 .^{\circ}$ & Luis & 1,2 & Luisa & 0,4 \\
\hline $16 .^{\circ}$ & Domingo & 1,1 & Marina & 0,4 \\
\hline $17 .^{\circ}$ & Álvaro & 1,0 & Águeda & 0,4 \\
\hline $18 .^{\circ}$ & Lorenzo & 0,9 & Antonia & 0,4 \\
\hline $19 .^{\circ}$ & Miguel & 0,9 & Brianda & 0,4 \\
\hline $200^{\circ}$ & Antonio & 0,9 & Cecilia & 0,4 \\
\hline $21 .^{\circ}$ & Sebastián & 0,7 & Felipa & 0,4 \\
\hline $22 .^{\circ}$ & Tomé-Tomás & 0,7 & Guiomar & 0,4 \\
\hline $23 .^{\circ}$ & Benito & 0,7 & Jerónima & 0,4 \\
\hline $24 .^{\circ}$ & Gaspar & 0,6 & Mariana & 0,4 \\
\hline $25 .^{\circ}$ & Gómez & 0,6 & María Isabel & 0,4 \\
\hline $26 .^{\circ}$ & Jerónimo & 0,6 & Olalla & 0,4 \\
\hline $27 .^{\circ}$ & Mateo & 0,6 & Lisenda & 0,4 \\
\hline $28^{\circ}$ & Sancho & 0,6 & & \\
\hline $29 .^{\circ}$ & Manuel & 0,5 & & \\
\hline $30{ }^{\circ}$ & Melchor & 0,5 & & \\
\hline $31 .^{\circ}$ & Baltasar & 0,5 & & \\
\hline $32 .^{\circ}$ & Lope & 0,5 & & \\
\hline $33^{\circ}$ & Blas & 0,5 & & \\
\hline $34 .^{\circ}$ & Fabián & 0,4 & & \\
\hline $35 .^{\circ}$ & Esteban & 0,3 & & \\
\hline $36 .^{\circ}$ & Marcos & 0,3 & & \\
\hline $37 . \circ$ & Nuño & 0,3 & & \\
\hline $38 .^{\circ}$ & Ruy & 0,3 & & \\
\hline $39 .^{\circ}$ & Gabriel & 0,3 & & \\
\hline $400^{\circ}$ & Vasco & 0,3 & & \\
\hline $41^{\circ}$ & Bernardino & 0,2 & & \\
\hline $42 .^{\circ}$ & Gil & 0,2 & & \\
\hline $43^{\circ}$ & Pascual & 0,2 & & \\
\hline $44 .^{\circ}$ & Santos & 0,2 & & \\
\hline $45 .^{\circ}$ & Simón & 0,2 & & \\
\hline $46 .^{\circ}$ & Agustín & 0,1 & & \\
\hline $47 .^{\circ}$ & Gregorio & 0,1 & & \\
\hline $48^{\circ}$ & Jorge & 0,1 & & \\
\hline $49 .^{\circ}$ & Lucas & 0,1 & & \\
\hline $500^{\circ}$ & Pablo & 0,1 & & \\
\hline $51 . \circ$ & Alejo & 0,1 & & \\
\hline $52 .^{\circ}$ & Arias & 0,1 & & \\
\hline $53^{c}$ & Bernal & 0,1 & & \\
\hline $54 .^{\circ}$ & Gutierre & 0,1 & & \\
\hline $55 .^{\circ}$ & Iñigo & 0,1 & & \\
\hline
\end{tabular}


Extremeños pasajeros a Indias en el siglo xVI (continuación)

\begin{tabular}{|c|c|c|c|c|}
\hline \multirow[b]{2}{*}{ N. de orden } & \multicolumn{2}{|c|}{ Varones } & \multicolumn{2}{|c|}{ Mujeres } \\
\hline & Nombres & Frecuencia (\%) & Nombres & Frecuencia (\%) \\
\hline 56. & Nicolás & 0,1 & & \\
\hline $57 .^{\circ}$ & Salvador & 0,1 & & \\
\hline $58 .^{\circ}$ & Aparicio & & & \\
\hline $59 .^{\circ}$ & Carlos & & & \\
\hline $60 .^{\circ}$ & Zebrián & & & \\
\hline $61 .^{\circ}$ & Esplandián & & & \\
\hline $62 .^{\circ}$ & Felipe & & & \\
\hline $63 .^{\circ}$ & Ginés & & & \\
\hline $64 .^{\circ}$ & Isidoro & & & \\
\hline $65 .^{\circ}$ & Juan Pablo & & & \\
\hline $66^{\circ}$ & Justo & & & \\
\hline $67 .^{\circ}$ & Leonel & & & \\
\hline $68 .^{\circ}$ & Matias & & & \\
\hline $69 .^{\circ}$ & Nazario & & & \\
\hline $70^{\circ}$ & Nuño & & & \\
\hline $71 .^{\circ}$ & Pedro Miguel & & & \\
\hline $72 .^{\circ}$ & Roque & & & \\
\hline $73 .^{\circ}$ & Santiago & & & \\
\hline $74 .^{\circ}$ & Tristán & & & \\
\hline $75^{\circ}$ & Valentín & & & \\
\hline
\end{tabular}

El análisis cuantitativo nos da los siguientes valores:

Extremeños viajeros a Indias en el siglo xvI

\begin{tabular}{|c|c|c|}
\hline Índices & Mujeres & Hombres \\
\hline N. nombres & 27 & 75 \\
\hline N. ${ }^{\circ}$ personas & 255 & 2.073 \\
\hline Nombres usados por más de una persona & $16(59 \%)$ & $57(76 \%)$ \\
\hline Nombres usados sólo por una persona & $11(41 \%)$ & $18(24 \%)$ \\
\hline Dispersión & 0,106 & 0,036 \\
\hline PI & 9,44 & 27,64 \\
\hline INU & 0,41 & 0,024 \\
\hline II & 0,351 & 0,555 \\
\hline
\end{tabular}

Estos valores están muy influidos por la diferencia de magnitud de la relación de hombres (2.073 personas), respecto de la de mujeres (255), pero a diferencia de la de los ilegítimos de Cáceres y en correspondencia con las de Mérida se evidencia entre las mujeres la tendencia a acentuar su individualización mediante el uso de nombres unipersonales. Tampoco aquí aparece el nombre de Bernabé. 
Otra relación de nombres es la de los asistentes al Sínodo diocesano de Badajoz del año $1565^{16}$, lógicamente sólo de varones, y es la siguiente:

Asistentes al Sínodo Diocesano de Badajoz de 1565

\begin{tabular}{|c|c|c|}
\hline$N^{\circ}$ orden & Nombre & Frecuencia (\%) \\
\hline $1 .^{\circ}$ & Juan & 16,8 \\
\hline $2 .^{\circ}$ & Francisco & 12,4 \\
\hline $3 .^{\circ}$ & Alonso & 10,1 \\
\hline $4 .^{\circ}$ & Rodrigo & 7,9 \\
\hline $5 .^{\circ}$ & Pedro & 6,7 \\
\hline $6 .^{\circ}$ & Gonzalo & 6,7 \\
\hline $7 .^{\circ}$ & Diego & 4,5 \\
\hline $8 .^{\circ}$ & Fernando & 4,5 \\
\hline $9 .^{\circ}$ & Cristóbal & 3,4 \\
\hline $10^{\circ}$ & Gómez & 3,4 \\
\hline $11 .^{\circ}$ & Álvaro & 2,2 \\
\hline $12 .^{\circ}$ & Antonio & 2,2 \\
\hline $13 .^{\circ}$ & Baltasar & 2,2 \\
\hline $14 .^{\circ}$ & Benito & 2,2 \\
\hline $15 .^{\circ}$ & Hernán & 2,2 \\
\hline $16 .^{\circ}$ & Ruy & 2,2 \\
\hline $17 .^{\circ}$ & Bartolomé & 1,1 \\
\hline $18 .^{\circ}$ & Blas & 1,1 \\
\hline $19 .^{\circ}$ & García & 1,1 \\
\hline $200^{\circ}$ & Jorge & 1,1 \\
\hline $21 .^{\circ}$ & Lope & 1,1 \\
\hline $22 .^{\circ}$ & Lorenzo & 1,1 \\
\hline $23 .^{\circ}$ & Martín & 1,1 \\
\hline $24 .^{\circ}$ & Nicolás & 1,1 \\
\hline $25 .^{\circ}$ & Vasco & 1,1 \\
\hline
\end{tabular}

El análisis cuantitativo nos proporciona los siguientes datos:

\begin{tabular}{lc}
\hline \multicolumn{1}{c}{ Concepto } & Hombres \\
\hline N. ${ }^{\circ}$ nombres & 25 \\
N. ${ }^{\circ}$ persona & 89 \\
Nombres usados por más de una persona & $16(64 \%)$ \\
Nombres usados sólo por una persona & $9(36 \%)$ \\
Dispersión & 0,28 \\
PI & 3,56 \\
INU & 0,37 \\
II & 0,41 \\
\hline
\end{tabular}

16 E. MÉndez VEnEGAS: “Asistencia a los sinodos diocesanos de 1568 y 1640 ». Revista de Estudios Extremeños. Tomo XL, Núm. I (Badajoz, 1984), págs. 161-170. 
Es evidente que los nombres de estos clérigos, que en el momento de su bautismo se desconocia llegarían a ese estado, responden a la tendencia general que vemos en otros lugares de Extremadura, tanto en la variedad de nombres, en su ordenación según la proporción de su asignación y en la relación entre nombres pluripersonales y unipersonales.

Finalmente disponemos de un acta de confirmación sacramental en Llerena del año $1576^{17}$, de la cual hemos extraído las siguientes relaciones, que exponemos ordenadas de mayor a menor frecuencia relativa:

Confirmaciones en Llerena en 1576

\begin{tabular}{|c|c|c|c|c|}
\hline \multirow[b]{2}{*}{ N. de orden } & \multicolumn{2}{|c|}{ Niños } & \multicolumn{2}{|c|}{ Niñas } \\
\hline & Nombres & Frecuencia (\%) & Nombres & Frecuencia (\%) \\
\hline $1 .^{\circ}$ & Juan & 22,2 & María & 38,6 \\
\hline $2 .^{\circ}$ & Alonso & 14,3 & Isabel & 15,3 \\
\hline $30^{\circ}$ & Francisco & 13,5 & Catalina & 9,2 \\
\hline $4 .^{\circ}$ & Pedro & 10,3 & Ana & 6,1 \\
\hline $5 .^{\circ}$ & Cristóbal & 6,3 & Francisca & 6,1 \\
\hline $6 .^{\circ}$ & Diego & 4,8 & Beatriz & 5,3 \\
\hline $7 .^{\circ}$ & Antonio & 3,2 & Leonor & 3,8 \\
\hline $8 .^{\circ}$ & Miguel & 2,4 & Juana & 1,5 \\
\hline $9 .^{\circ}$ & Gaspar & 2,4 & Lorenza & 1,5 \\
\hline $10^{\circ}$ & Bartolomé & 2,4 & Inés & 1,5 \\
\hline $11 .^{\circ}$ & Hernando & 1,6 & Cecilia & 1,5 \\
\hline $12^{\circ}$ & Jerónimo & 1,6 & Gracia & 0,8 \\
\hline $13 .^{\circ}$ & Gonzalo & 1,6 & Costanza & 0,8 \\
\hline $14 .^{\circ}$ & Agustín & 1,6 & Lucía & 0,8 \\
\hline $15 .^{\circ}$ & Luis & 1,6 & Madalena & 0,8 \\
\hline $16 .^{\circ}$ & Martín & 1,6 & Elena & 0,8 \\
\hline $17 .^{\circ}$ & Benito & 1,6 & Andrea & 0,8 \\
\hline $18 .^{\circ}$ & Gutierre & 0,8 & Luisa & 0,8 \\
\hline $19 .^{\circ}$ & Sebastián & 0,8 & Maria Isabel & 0,8 \\
\hline $20^{\circ}$ & Blas & 0,8 & Mariana & 0,8 \\
\hline $21^{\circ}$ & Feliciano & 0,8 & Jerónima & 0,8 \\
\hline $22 .^{\circ}$ & Andrés & 0,8 & Teresa & 0,8 \\
\hline $23^{\circ}$ & Bernardino & 0.8 & & \\
\hline $24 .^{\circ}$ & Garcia & 0,8 & & \\
\hline $25 .^{\circ}$ & Claros & 0,8 & & \\
\hline $26 .^{\circ}$ & Bernabé & 0,8 & & \\
\hline
\end{tabular}

17 Libro de Asiento y autos de todas las personas que se confirman en la lglesia Mayor de Nra. Sra. de Llerena. Transcrito por José Luis Garrain Villa en "Documentos sobre Luis Zapata de Cárdenas y otros evangelizadores llerenenses en el Archivo de Llerena". Actas del congreso: "Extremadura en la evangelización del Nuevo Mundo", Guadalupe, año 1988. Ed. de Fr. Sebastián Garcia, OFM. Madrid, 1990, págs. 379-400. 
El resumen cuantitativo es el siguiente correspondiente es el que mostramos en el siguiente cuadro:

Confirmaciones en Llerena en 1578

\begin{tabular}{|c|c|c|}
\hline Indices & Niñas & Niños \\
\hline N. ${ }^{\circ}$ nombres & 22 & 26 \\
\hline N. ${ }^{\circ}$ personas & 126 & 131 \\
\hline Nombres usados por más de una persona & $12(55 \%)$ & $17(65 \%)$ \\
\hline Nombres usados sólo por una persona & $10(45 \%)$ & $9(35 \%)$ \\
\hline Dispersión & 16,79 & 20,63 \\
\hline $\mathrm{PI}$ & 5,95 & 4,85 \\
\hline INU & 0,55 & 0,346 \\
\hline II & 0,298 & 0,427 \\
\hline
\end{tabular}

En total son 257 los confirmados, de los cuales $18(7 \%)$ niños repiten el nombre de su padre, 3 niñas repiten el nombre de su padre $(1,2 \%)$ y 1 el de su madre $(0,4 \%)$, lo que confirma la tendencia mayoritaria a repetir la nominalidad paterna.

Lo hasta aqui visto de las nominaciones utilizadas por los extremeños nos muestra una gran homogeneidad, pues entre las mujeres siempre es María el nombre más utilizado, en torno al $40 \%$, seguido de Isabel (11\%), Catalina (9\%), Ana, Inés, Juana y Beatriz, conjunto de nombres que cubren el $80 \%$ del total de las personas. Entre los varones también se produce unanimidad entre los siete primeros, que vienen a nominar al $70 \%$ de ellos, siempre encabezados por Juan $(20 \%)$, Francisco (13\%), Alonso (10\%), Pedro, Diego, Hernando y Gonzalo.

Sin embargo, si comparamos estas conclusiones con una lista que proporciona Rodríguez Moñino ${ }^{18}$ de los procesados en Badajoz, acusados de judaizantes, observaremos algunas diferencias, las relaciones son las siguientes.

Procesados acusados de judaizantes en Badajoz en el siglo xvI

\begin{tabular}{c|lc|lc}
\hline \multirow{2}{*}{ N. de orden } & \multicolumn{2}{|c|}{ Varones } & \multicolumn{2}{c}{ Mujeres } \\
\cline { 2 - 5 } & Nombres & Frecuencia (\%) & Nombres & Frecuencia (\%) \\
\hline $1 .^{\circ}$ & Diego & 15,0 & Catalina & 14,4 \\
$2 .^{\circ}$ & Francisco & 12,9 & Isabel & 13,6 \\
$3 .^{\circ}$ & Juan & 12,4 & Beatriz & 12,7 \\
$4 .^{\circ}$ & Alonso & 8,6 & Inés & 8,5 \\
$5 .^{\circ}$ & Fernando & 7,5 & María & 8,5 \\
6. & Manuel & 7,0 & Leonor & 7,6 \\
\hline
\end{tabular}

18 A. Rodriguez Moñino: «Les judaisants a Badajoz de 1493 a 1599 ". Revue des Etudes Juives. Tomo CXV (1956), págs. 73-86. 
Procesados acusados de judaizantes en Badajoz en el siglo XVI (continuación)

\begin{tabular}{|c|c|c|c|c|}
\hline \multirow[b]{2}{*}{$N^{\circ}$ de orden } & \multicolumn{2}{|c|}{ Varones } & \multicolumn{2}{|c|}{ Mujeres } \\
\hline & Nombres & Frecuencia (\%) & Nombres & Frecuencia (\%) \\
\hline $7 .^{\circ}$ & Gonzalo & 4,8 & Ana & 4,2 \\
\hline $8 .^{\circ}$ & Rodrigo & 4,3 & Gracia & 4,2 \\
\hline $9 .^{\circ}$ & Pedro & 3,8 & Mayor & 4,2 \\
\hline $10 .^{\circ}$ & Simón & 2,1 & Elvira & 3,4 \\
\hline $11 .^{\circ}$ & Hernando & 2,1 & Blanca & 2,5 \\
\hline $12 .^{\circ}$ & Enrique & 1,6 & Guiomar & 2,5 \\
\hline $13 .^{\circ}$ & García & 1,6 & Juana & 2,5 \\
\hline $14 .^{\circ}$ & Lope & 1,6 & Elena & 1,7 \\
\hline $15 .^{\circ}$ & Nuño & 1,1 & Costanza & 0,8 \\
\hline $16 .^{\circ}$ & Sebastián & 1,1 & Engracia & 0,8 \\
\hline $17 .^{\circ}$ & Gabriel & 1,1 & Felipa & 0,8 \\
\hline $18 .^{\circ}$ & Álvaro & 1,1 & Francisca & 0,8 \\
\hline $19 .^{\circ}$ & Duarte & 1,1 & Marina & 0,8 \\
\hline $20 .^{\circ}$ & Gaspar & 1,1 & Mencía & 0,8 \\
\hline $21 .{ }^{\circ}$ & Lorenzo & 1,1 & Olalla & 0,8 \\
\hline $22 .^{\circ}$ & Benito & 1,1 & Sara & 0,8 \\
\hline $23 .^{\circ}$ & Antonio & 1,1 & Susana & 0,8 \\
\hline $24 .^{\circ}$ & Tomás & 0,5 & Teresa & 0,8 \\
\hline $25 .^{\circ}$ & Blasco & 0,5 & Violante & 0,8 \\
\hline $26 .^{\circ}$ & Ruy & 0,5 & & \\
\hline $27 .^{\circ}$ & Martín & 0,5 & & \\
\hline $28 .^{\circ}$ & Bartolomé & 0,5 & & \\
\hline $29 .^{\circ}$ & Suero & 0,5 & & \\
\hline $30 .^{\circ}$ & Baltasar & 0,5 & & \\
\hline 31. & Miguel & 0,5 & & \\
\hline $32 .^{\circ}$ & Salvador & 0,5 & & \\
\hline $33 .^{\circ}$ & David & 0,5 & & \\
\hline $34 .^{\circ}$ & Gómez & 0,5 & & \\
\hline $35 .^{\circ}$ & Antón & 0,5 & & \\
\hline $36^{\circ}$ & Vasco & 0,5 & & \\
\hline
\end{tabular}

Estas relaciones sí muestran divergencias apreciables con todas las anteriores; como más llamativa, e, indudablemente, significativa es la posición de Maria entre los nombres femeninos, la $4{ }^{a}-5 .^{a}$ en la ordenación de mayor a menor y con una aplicación sobre la población del $8,5 \%$, es decir, la quinta parte que en cualquier otra; este hecho no debía ser una excepción en la conducta de los judeoconversos, pues en un árbol genealógico que muestra Caro Baroja ${ }^{19}$, los nombres de mujeres son Leonor ( 7 veces), Beatriz (6), Teresa (3), Isabel, María Catalina y Juana (2 ve-

19 J. Caro Baroja: Los judíos en la España Moderna y Contemporánea. Tomo I. Madrid, 1978, págs. 418 y 419. 
ces cada una), y una sóla vez los de Gracia, Ana, Flora, Elvira y Guiomar, mientras que la de los varones está encabezada por Juan (6 veces), Pedro (5), Diego (4), Francisco (3), Álvaro y Hernando (2), Andrés, Antonio, Martín y Alonso (1 vez cada uno); también en la lista de nombres aparecen aunque sólo asignadas a una persona cada nombre, los de Sara y Susana; como igualmente vemos con el nombre David con los varones, entre los que aparece el nombre de Duarte, de uso tan extendido en Portugal, como el de Enrique; hay que recordar que, a partir de 1580 , cuando Portugal se incorpora a la Monarquía Hispánica, muchos marranos portugueses regresaron a Castilla. Por otra parte, tampoco vemos entre los nombres de varones el de Bernabé.

El cuadro estadístico para los judaizantes es el siguiente:

Judaizantes en Badajoz

\begin{tabular}{lcc}
\hline \multicolumn{1}{c}{ Indices } & Hombres & Mujeres \\
\hline N. ${ }^{\circ}$ nombres & 36 & 25 \\
N. ${ }^{\circ}$ personas & 186 & 118 \\
Nombres usados por más de una persona & $23(63,89 \%)$ & $14(56 \%)$ \\
Nombres usados sólo por una persona & $13(36,11 \%)$ & $11(44 \%)$ \\
Dispersión & 0,19 & 021 \\
PI & 5,17 & 4,72 \\
INU & 0,36 & 0,44 \\
II & 0,408 & 0,314 \\
\hline
\end{tabular}

La historia de las mentalidades, como decia Fernand Braudel, hay que estudiarla dentro de los procesos de larga duración, por ser los menos cambiantes de los diversos aspectos y formas que configuran una sociedad determinada, pero también permiten muchas vías de aproximación a su conocimiento, y entre ellas hemos ensayado esta de la onomástica, difícil de interpretar, no sólo por el carácter polisémico que adquieren muchos nombres, tanto por su etimología, el ámbito político-cultural en el que se ha desarrollado y desde el que ha sido transferido a otros, las connotaciones étnicas, religiosas, o de cualquier otra índole; como, por otra parte, lo que cada nombre significa en ámbitos más reducidos, las familias, o las localidades. No obstante, este acercamiento tan elemental como el que hemos realizado nos ha permitido ver cómo, dentro de unas sociedades de las que pensamos evolucionaban muy lentamente, tanto por las características de su tiempo como del propio ambiente del territorio, se manifiestan a través de la asignación del nombre a los niños al bautizarlos, unas atracciones, o unos rechazos, hacia unos nombres $u$ otros, lo que indica que unas veces se pretende manifestar la adhesión a una figura determinada, otras lo que se quiere es diluir la propia identidad familiar dentro de un grupo dominante, mientras que, en otros casos, se quiere evidenciar que uno pertenece a un grupo determinado que no acepta valores significativos de 
la sociedad dominante (el poco uso de los judaizantes del nombre de María) y que además asume su propia identidad étnica recogiendo nombres característicos de la misma (David, Sara).

El conocimiento de la onomástica, como sistema de símbolos representativos de creencias y, a la vez, como hemos visto, como imágenes con las que los individuos quisieran ser vistos por el conjunto de la sociedad, que al responder a intenciones y causalidades distintas nos aleja de la idea generalizada de que los nombres se asignan por criterios de continuidad de una identidad o tradición familiar, y en última instancia como una muestra de religiosidad, puede ser un procedimiento aceptable para contribuir a la historia de las mentalidades, porque cumple algunos de los requisitos que Peter Burke ${ }^{20}$ establece para la misma, porque refleja actitudes colectivas en lugar de las individuales, $y$, por otra parte, comprende los comportamientos de la población en su totalidad, y no solamente la de un grupo determinado.

La Onomástica también nos puede dar alguna información respecto a lo que en ella se refleje por parte de grupos de cultura alta, élites, o grupos de cultura popular; igualmente en cuanto a la prelación de nombres dentro de las familias conforme se van bautizando los hijos, o cómo se refleja la onomástica de los abuelos en los nietos, o en los nacimientos de esclavos si reproducen los nombres de sus propietarios o los de sus progenitores; igualmente, la aparición de nombres nuevos en localidades y regiones determinadas nos pueden señalar la llegada de inmigrantes de otras regiones diferentes, y recíprocamente, la desaparición de algunos nombres también podrá relacionarse con corrientes de emigrantes, son todos ellos aspectos de gran interés y que ofrecen muchas posibilidades para conocer cambios demográficos y sociales, cuestiones que por la limitación de espacio no abordamos en este artículo.

También sería deseable que, al cubrir nuestra Universidad todo el territorio nacional, pudiésemos disponer de trabajos de demografía y onomástica que nos proporcionaran el conocimiento más amplio posible de la sociedad española del Antiguo Régimen.

20 P. Burke: Formas de historia... Madrid, 1999, págs. 207-230. 